\title{
lodine-125 brachytherapy for brain tumours - a review
}

\author{
Silke B Schwarz ${ }^{1 *}$, Niklas Thon², Katharina Nikolajek ${ }^{1}$, Maximilian Niyazi ${ }^{1}$, Joerg-Christian Tonn², Claus Belka ${ }^{1}$ and \\ Friedrich-Wilhelm Kreth ${ }^{2}$
}

\begin{abstract}
lodine-125 brachytherapy has been applied to brain tumours since 1979. Even though the physical and biological characteristics make these implants particularly attractive for minimal invasive treatment, the place for stereotactic brachytherapy is still poorly defined.

An extensive review of the literature has been performed, especially concerning indications, results and complications. lodine-125 seeds have been implanted in astrocytomas I-III, glioblastomas, metastases and several other tumour entities. Outcome data given in the literature are summarized. Complications are rare in carefully selected patients.

All in all, for highly selected patients with newly diagnosed or recurrent primary or metastatic tumours, this method provides encouraging survival rates with relatively low complication rates and a good quality of life.
\end{abstract}

Keywords: Stereotactic brachytherapy, interstitial brachytherapy, seed, iodine-125, brain tumour

\section{Introduction}

The implantation of radioactive material into tumours was proposed by Pierre Curie in 1901 [1]. In 1951, Friedlander and Orr prepared iodine- 125 by alpha bombardment of natural antimony [2]. In 1965, this nuclide was finally introduced in interstitial cancer therapy (prostate, lung cancer and lymph nodes) [3]. Mundinger played a pioneering role in the development of the concept of brachytherapy in brain tumours: he began to implant iridium-192 wires into gliomas at 1960; since 1979 he has preferred iodine-125 seeds [4,5]. Nowadays, temporary implantation of iodine- 125 seeds is preferred for interstitial brachytherapy in brain tumours. Despite favourable physical and biological characteristics of brachytherapy and well established implantation techniques, so far brachytherapy treatment of brain tumours has been performed in only a few centres worldwide. The current review considers radiobiological characteristics, indications, and treatment effects of iodine-125 brachytherapy (including side effects) for patients with brain tumours.

\footnotetext{
* Correspondence: Silke.Schwarz@med.uni-muenchen.de

'Department of Radiation Oncology, Ludwig-Maximilians-University Hospital, Marchioninistr. 15, 81377 Munich, Germany

Full list of author information is available at the end of the article
}

\section{Review}

Rationale for stereotactic iodine-125 brachytherapy

Interstitial brachytherapy aims for a highly localized devitalisation of a well defined treatment volume, thereby avoiding damage of the surrounding non-neoplastic tissue. Treatment volume and target volume are ideally identical. The minimal-invasive, spatially precise stereotactic implantation technique, in combination with favourable physical characteristics of the radioactive sources, enable the accurate application of highly focused necrotizing tissue dose with a steep fall-off from the centre to the periphery. The pronounced dose inhomogeneity within the target volumes make iodine- 125 brachytherapy an attractive concept, particularly for selected patients suffering from small, circumscribed tumours at any location of the brain. Of note, stereotactic brachytherapy must not be confused with stereotactic radiotherapy, which exhibits much less dose inhomogeneity and lower intratumoural (non-necrotizing) doses, and stereotactic radiosurgery, which is characterized by the absence of any effects of fractionation [6-10].

\section{Physical characteristics, dosage and dosimetry}

Conventional fractionated irradiation is administered with a dose rate of about $200 \mathrm{cGy} / \mathrm{min}$. In contrast, interstitial 
irradiation aims for much lower dose rates of $<100 \mathrm{cGy} / \mathrm{h}$. This continuous low-dose rate irradiation increases the therapeutic ratio as the ongoing repair of sublethal irradiation doses is more effective in non-neoplastic tissue, than in tumour (see below).

Iodine-125 emits gamma-rays with a very low average photon energy of $28.5 \mathrm{keV}[11,12]$, has a specific gammaray constant of $1.32-1.45 \mathrm{R} \cdot \mathrm{cm}^{2} / \mathrm{mCi} \cdot \mathrm{h}$ [11], a long half-life of 59.4-60.2 days [2,12-14], a half-value tissue thickness of $2 \mathrm{~cm}$ and for lead of $0.025 \mathrm{~mm}$, which enables easy shielding $[4,15]$. Iodine- 125 implants generate a typically extreme dose inhomogeneity within target volumes that ranges from highly necrotizing doses surrounding the seeds to the ultimate form of fractionation at the periphery of target volumes [16] (Table 1).

There are two forms of iodine-125 brachytherapy techniques: temporary and permanent [17] (Table 2). Currently, temporary implants are preferred as permanent implants bear an increased risk of prolonged oedema [18-20]. Exclusively low activity iodine-125 seeds (< $20 \mathrm{mCi}$ ) are preferred for slowly proliferating processes, such as low-grade gliomas, to achieve a dose of 50-60Gy at the tumour margin; the dose rate is usually extremely low (10cGy/h; range 5-20cGy/h). High-dose-rate brachytherapy (> 30cGy/h), as being achieved with high activity seeds of up to $40 \mathrm{mCi}$, however, may be required for rapidly-proliferating high-grade gliomas [12,21,22]. Of note, high dose rates (30-60cGy/h) alone or in combination with external beam radiation have been shown to be associated with a high frequency of radiogenic complications (30-50\%) [23-25].

To calculate the total dose for permanent iodine- 125 implants the mean life time of 87 days is used [26]. As compared with alternative permanent implants (Au-198 and Pd-103), I-125 has been shown to be more effective on slow growing tumours (tumour doubling time of $>10$ days) [27].

Principles of seed positioning aim for a good coverage of the target volumes with an acceptable uniformity of the dose distribution by a minimal number of catheter implants while sparing the surrounding anatomical structures (i.e. normal brain tissue, vessels, optic nerve etc.) from radiation exposure [28-33]. Usually, one to five seeds, encapsulated in the tip of a Teflon catheter, are

Table 1 Isotopes used for brachytherapy in brain tumours (Adapted from [16]).

\begin{tabular}{llll}
\hline Isotope & Emission & Mean energy (MeV) & Half-life (days) \\
\hline lodine-125 & $\gamma$ & 0.028 & 60 \\
\hline Iridium-192 & $\gamma$ & 0.38 & 74 \\
\hline Phosphorus-32 & $\beta$ & 0.69 & 14 \\
\hline Rhenium-186 & $\beta$ & 0.36 & 4 \\
\hline Yttrium-90 & $\beta$ & 0.93 & 3 \\
\hline
\end{tabular}

Table 2 Parameters of permanent and temporary iodine125 seeds (Adapted from [13,147]).

\begin{tabular}{lll}
\hline & Permanent seeds & Temporary seeds \\
\hline Activity per source & $0.1-3 \mathrm{mCi}$ & $3-50 \mathrm{mCi}$ \\
\hline Dose & $80-700 \mathrm{~Gy}$ & $30-200 \mathrm{~Gy}$ \\
\hline Dose rate & $3-30 \mathrm{cGy} / \mathrm{h}$ & $3-125 \mathrm{cGy} / \mathrm{h}$ \\
\hline Number of sources per case & $1-171$ & $1-28$ \\
\hline Time to dose & infinity & $4-50$ days \\
\hline
\end{tabular}

necessary to achieve a conformal interstitial irradiation, even of complexly shaped tumour volumes. Of note, iodine- 125 seeds are available in various shapes, making their dose distribution non-isotropic, which must be considered for therapy planning [11,14,34-40]. The tri-planar treatment-planning system is based on stereotactic computed tomography $(\mathrm{CT})$ and magnetic resonance imaging (MRI), as well as novel metabolic imaging data. It allows positioning of seeds within target volumes, generate and display the resulting isodose distribution and calculate approach angles $[32,41,42]$. Care has to be taken that the high dose zones ( $>150 \mathrm{~Gy}$ ) always lie within the tumour tissue and that vessels are not adjacent to these zones [43].

The definition of target volumes has changed over time. In the beginning of seed implantation in brain tumours, the target volume was mostly determined to be the tumour visible in CT, plus an isotropic margin of $0.5 \mathrm{~cm}$ [23,30,44-47]. Presently, image fusion with magnetic resonance imaging and positron emission tomography allows a better visualisation and more precise definition of the target volume [16]. Schupak et al. found out that the technical accuracy had an impact on the local failure rate but not on the overall survival of the patients [48].

The following parameters should be documented for each stereotactic brachytherapy: prescribed total dose, dose rate, minimum tumour dose, percentage of the tumour receiving less than the prescribed dose, maximum dose in "normal" brain at $1 \mathrm{~cm}$ from the tumour border, number of seeds, activity of seeds, volumes of tumour and surrounding tissue irradiated to various total doses and dose rates [49].

In summary, the accurate dose distribution, the rapid dose decrease from the centre of the treatment volume towards the periphery, and the high doses in the tumour and the low doses in the normal tissue, are great physical advantages of iodine-125 implants for brain tumour therapy [16].

\section{Biological characteristics \\ Theoretical models and cell culture experiments}

Dale et al. have adapted the linear-quadratic (LQ) model for protracted radiotherapy, incorporated tumour repopulation and tumour shrinkage factors, to allow 
radiobiological assessment of permanently implanted iodine-125 seeds [50-52]. Using the conventional linearquadratic model with an $\alpha / \beta=10$ Gy for tumour tissue and an $\alpha / \beta=3 \mathrm{~Gy}$ for healthy tissue and given a repopulation resulting in a loss of $0.4 \mathrm{~Gy} / \mathrm{d}, 60 \mathrm{~Gy}$ of temporary implants in 5 days are almost equivalent to 100Gy permanent implant dose [53].

Even though the conventional LQ model describes well the radiobiological effects of an implant at the boundary of the target volume, it does not account for the effects of extreme dose inhomogeneity associated with brachytherapy [54]. Characteristic tissue effects associated with the high dose zone in the vicinity of the implanted source ( $\geq 200 \mathrm{~Gy}$ ) have been described experimentally, i. e. the development of circumscribed radionecrosis with temporary changes in capillary permeability with a sometimes extensive oedema and concomitantly reduced regional cerebral blood flow [18-20,55,56] (see below).

The relative biological effectiveness (RBE) of iodine-125 compared to Cobalt-60 is 1.3 to 1.5 [57-59]. Compared to iridium-192, iodine-125 has a RBE of 1 [60].

The overall low-dose-rate of iodine- 125 allows reoxygenation for increasing radiosensitivity and cancels out the repair of sublethal damage. Normal tissue irradiation is essentially reduced because the exposure level decreases by the inverse square of the distance from the source. Therefore, the rate of tumour cell killing of brachytherapy has been thought to be much greater than that of conventionally fractionated teletherapy [22].

\section{Animal experiments and histological studies}

Iodine-125 brachytherapy reduces histological features that are prognostic for tumour progression, i.e. cellularity, pleomorphism, vessel hyperplasia and degree of mitosis, and lowers the proliferating cell nuclear antigen, a marker for late G1- and S-phases of the cell cycle [61].

To examine how the tumour microenvironment (perfusion, oxygen partial pressure) changes in response to lowdose-rate brachytherapy, experiments with a mouse hypoxic tumour model have been performed. The perfusion is elevated at a distance of $2-4 \mathrm{~mm}$ from the seed, starting three days after implantation, and $\mathrm{pO}_{2}$ is increased one to three days after implantation. Therefore, additional external beam radiotherapy should be most advantageous 1-2 days after implantation, when $\mathrm{pO} 2$ is high, and chemotherapy most advantageous 3-4 days after implantation, when perfusion is high [62].

Autopsy material showed that the early phase after implantation is characterized by migrating macrophages and removal of necrotic debris. In the established phase, a necrotic centre and a reactive zone around have been found. The reactive zone consists of a narrow inner rim of microglial accumulation and a broader outer area with astrocytic gliosis, vascular proliferation, activated microglia and infiltration by macrophages. In the burned-out phase the necrosis undergoes liquefaction, the microglia rim is replaced by end-stage macrophages and the reactive zone is transformed into astrocytic gliosis equivalent to a scar $[55,63]$.

A radiomorphological triple ring formation has been identified to be characteristic after seed implantation (see below) and correlates with histopathological alterations including a coagulative necrosis in the central radiolucent region, a fibrinoid necrosis of vessel walls within the ring enhancement and an adjacent spongiosis and gliosis, as well as oedema [18]. With increasing time after implantation the ring enhancement moves outward [64].

The effect of permanent iodine-125 implants on the blood-brain barrier (BBB) function was additionally studied in normal canine brains. The breakdown of the BBB occurs as soon as 7 days after implantation. The radiation lesion enlarges rapidly from day 7 to 72 , then increases more slowly until day 392 and is relatively decreased in size in 2 years. Furthermore, the finding of the three zones with calcified necrosis, a narrow fluid-filled zone and a narrow rim of viable but damaged tissue in which there is breakdown of the BBB for up to 1 year before returning to an almost normal function at 2 years, were confirmed. Increased permeability was most pronounced in white matter as compared to grey matter [19].

\section{Summary of biological characteristics}

Due to the continuous low-dose rate irradiation, especially in the periphery of the target volume and at the edge to normal tissue, sublethal damage can be repaired and long term side effects of the late-responding tissue can be avoided. In the centre of the treated volume, highly focused necrotizing intratumoural doses with a steep dose decrease from the centre to the periphery, such as in radiosurgery, can be achieved. Therefore, seeds combine the advantages of fractionated radiotherapy (repair of surrounding normal tissue) and radiosurgery (tumour cell death irrespective of radiosensitivity) in one modality.

Because repopulation and redistribution during the treatment are of minor importance in the therapy of low grade gliomas, low dose rate seed implantation appears to be a rational therapeutic strategy [16].

\section{Stereotactic biopsy and implantation techniques}

Obtaining tissue diagnosis and molecular genetic profiling are mandatory for each suspected brain tumour. This can be routinely performed by means of stereotactic biopsy procedures that integrate CT, MRI and metabolic imaging data for trajectory planning and enable minimalinvasive collection of representative specimens throughout entire tumour volumes (including biologically "hot spots" if present), especially for those tumours that are not eligible for gross open tumour resections $[16,65,66]$. Therefore, the authors promote a stage concept of interstitial brachytherapy after versatile tissue diagnosis and 
molecular genetic profiling of the brain tumours have been obtained.

Seed implantation procedure is a multidisciplinary treatment involving neurosurgeons, radiation oncologists, neuro-radiologists and physicists [16]. A detailed description of the stereotactic implantation technique has been given in several reports by Kreth et al. $[16,67,68]$. Seed implantation is usually performed under general anaesthesia. After attaching a stereotactic ring to the patient's head, CT scans with a localizing system mounted on the base ring are obtained. Then the computer based treatment planning process starts, including 3D image reconstruction, image fusion with magnetic resonance imaging and/ or positron emission tomography, finding the optimum seed localisations and trajectories based on the coordinate system of the frame. The implantation of the iodine- 125 seeds (length: $4.5 \mathrm{~mm}$ ) loaded Teflon catheters is performed via a $2 \mathrm{~mm}$ burr-hole for each catheter. CT-scan follow-up is performed one day after surgery and fused with the pre-operative localized CT to control the seed positions. A hospital stay of about three days is required for the implantation procedure. The level of radiation upon discharge is checked and documented by the physicist $[12,13,16,17,30,69-74]$ (Figure 1). Steroids should be administered routinely on the day of implantation and for three days postoperatively at a daily decreasing dose of 24 , 12, 8 and $4 \mathrm{mg}$ dexamethasone, respectively. For temporary implants, the skin is re-opened for seed-removal after 20-30 days under local anaesthesia (no stereotactical equipment needed).

\section{Indications for iodine-125 brachytherapy}

Iodine-125 brachytherapy is a valuable treatment option for patients with non-resectable, small, and circumscribed untreated tumours in any location of the brain, as well as local circumscribed recurrences after previously performed percutaneous radiotherapy and/or surgery [75]. Ideally, the diameter of the tumour should be smaller than $3 \mathrm{~cm}$. In case of larger tumour volumes, microsurgery (partial resection) might be combined with brachytherapy [17]. Of note, interstitial brachytherapy can be performed even in cases in which tolerance of the healthy brain tissue has been reached because of previous external irradiation and/ or stereotactic radiosurgery $[4,76]$. Implantation is also a valuable technique in certain paediatric patients $[77,78]$. For a long time, infratentorial masses, the hypothalamic region, the middle-inferior mesencephalon, the diencephalon, the pons, the corpus callosum and tumours with subependymal or transcallosal spread, involvement of the cerebellum, the brainstem or the deep thalamic regions, involvement of basal ganglia structures, sylvian or interhemispheric fissures, have been considered contraindicated sites for implantation. Furthermore, large tumours $>6 \mathrm{~cm}$ or with a tumour volume of $>120 \mathrm{ml}$, multifocal masses,

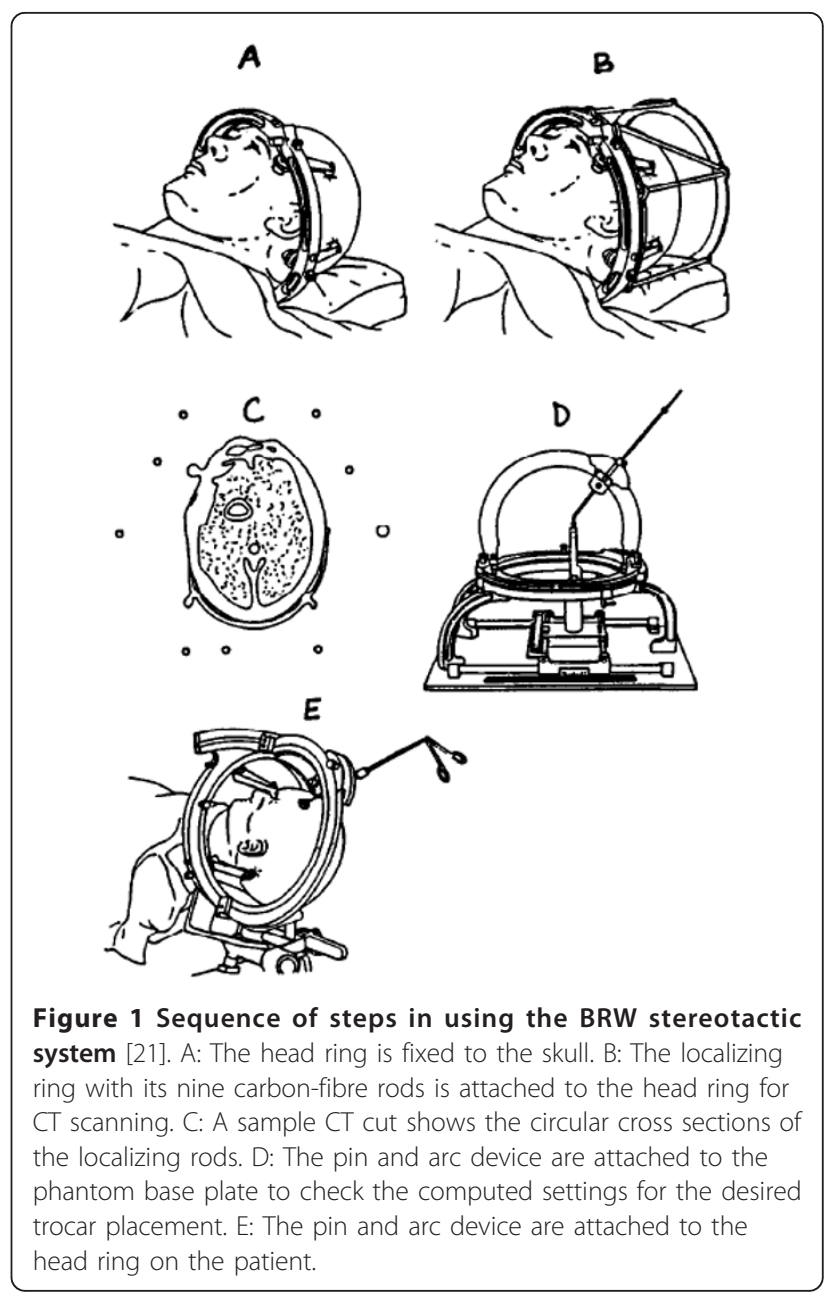

and those with diffuse margins, patients with a Karnofsky performance status (KPS) of $<60$ and significant oedema, have been thought to be not suitable for implantation [12,13,46,73,79-82]. Currently, technical indications have been extended $[83,84]$. Only diffuse tumours, tumours with a diameter larger than $4 \mathrm{~cm}$, or with infiltration of the corpus callosum, are still considered to be insuitable for implantation [16].

\section{Follow-up characteristics}

During MRI follow-up evaluation, a characteristic triple ring formation can be identified, which should not be confused with tumour progression [64]. The inner zone of this formation refers to the necrotized tumour, the intermediate contrast enhanced zone to a small rim of a still viable tumour, exhibiting increased capillary permeability, and the outer zone to the treatment induced oedema. The ring shaped area of contrast-enhancement is visible 4.5 months to 3 years after implantation [85]. A study on volumetric changes in tumour necrosis, reactive zone and oedema over the time after temporary seed implantation 
(50-60Gy) has revealed the following: compared to the reference dose volume the necrosis has been $19.9 \%$ after 6 months, $8.3 \%$ after 14 months and $7.0 \%$ after 24 months, the reactive zone $30.4 \%, 34.8 \%$ and $16.7 \%$, respectively, and the oedema $293.2 \%, 220.5 \%$ and $107.0 \%$, respectively. Necrosis has been found in regions with > 79.2Gy with a maximum 6 months after irradiation, a shrinkage between 9 and 15 months and a steady-state after 16 months. The reactive zone has had its maximum 2 months after irradiation, after 12 months the shrinkage has been more pronounced. The maximum of the oedema has been reached after 6 months [86].

Other visible late radiation effects ( $>2$ months) are decreased enhancement of the tumour region, decreased mass effect, atrophic changes in surrounding brain (widened sulci), calcification in areas immediately adjacent to the seeds, and focal necrosis corresponding to the treatment volume, surrounded by a zone of decreased cerebral metabolism in positron emission tomography (PET) [47].

To monitor iodine-125 brachytherapy effects in lowgrade gliomas with PET L-methyl-carbon-11-methionine uptake seems to be more suitable than 2-fluoro-18-1deoxy-D-glucose (FDG), as it shows a dose dependent decline in uptake $[87,88]$.

A magnetic resonance imaging study compared longitudinally features of patients either treated with external beam radiotherapy alone or in combination with brachytherapy. In both groups, nodular enhancement adjacent to or remote from the resection cavity strongly suggests tumour recurrence. Linear rim enhancement, later on progressing to feathery enhancement, immediately adjacent to the cavity, has been seen in brachytherapy patients only and strongly indicates radiation necrosis [89].

\section{Clinical indications and results}

The results concerning survival achieved with permanent and temporary brachytherapy implants in patients with various brain tumours are summarized in Table 3. For recurrent tumours, survival is measured starting from time of implantation. In the following section indications, clinical outcome, risk limits and side effects are given for different brain tumour entities.

\section{Brachytherapy of pilocytic astrocytomas WHO grade I}

Pilocytic astrocytomas frequently involve paediatric patients. Open tumour resection remains to be the gold standard, with favourable survival rates, provided complete tumour removal has been achieved. Unfortunately, these tumours are often found in highly eloquent locations such as the chiasmatic/hypothalamic area, the thalamus, the tractus opticus etc., rendering a complete resection impossible for a considerable number of patients. Poor physical, cognitive, and psychosocial outcome scores have been reported after long-term follow-up evaluation in particular for those patients with deep-seated tumour locations (diencephalon, optic chiasm, etc.). In here, an unfavourable outcome was at least partly attributed to side effects of surgical treatment [90-92].

In 1980, Mundinger et al. reported the first survival results of 55 young patients with diencephalic pilocytic astrocytomas treated with iodine-125 or iridium-192 interstitial brachytherapy. In this patient population 3- and 5year survival rates of $72 \%$ and $52 \%$ have been achieved [4]. In paediatric patients the 1-, 3- and 5-year survival rates were slightly better for permanent iridium-192 (88\%, 70\% and $55 \%)$ than for iodine- 125 implants $(86 \%, 58 \%$ and $22 \%)$. Both nuclides proved to be more effective than biopsy or partial resection only [93]. Others report a 2 -year survival rate of $83 \%$ for paediatric patients and temporary iodine-125 implants [94].

Another retrospective analysis revealed 1- and 3-year survival rates of $94 \%$ and $73 \%$ for newly diagnosed (treated in combination with percutaneous radiotherapy) and of $68 \%$ and $30 \%$ for recurrent (treated with brachytherapy alone) low grade gliomas [25].

The largest series with 106 pilocytic astrocytoma patients was published by Ostertag and Kreth. Patients were either treated with permanent or temporary implants; a 5-year survival rate of $77 \%$ was obtained and radiation toxicity was mostly observed in patients treated with permanent implants [95]. 5- and 10-year survival rates of up to $85 \%$ and $83 \%$ have been reported by another publication [68].

Of note, treatment of a subpopulation of 45 highly eloquent hypothalamic tumours was associated with low risk and provided similar clinical outcome rates. Peraud and co-workers report on clinical outcome after temporary iodine-125 implantation (54Gy, $8 \mathrm{cGy} / \mathrm{h},<20 \mathrm{mCi}$ ) for complex located WHO grade I and II gliomas (including 4 mesencephalic/pontine, 2 thalamic/pineal and 2 mesencephalic/pontine tumour locations) [72]. A complete response after brachytherapy was seen in four patients, and a partial response in seven patients. None of the patients exhibited tumour progression within a median follow-up of 31.5 months, and no radiogenic complications occurred. Of note, functional outcome scores were favourable with significant improvement of pre-existing hemiparesis in 3/4 patients, improvement of endocrine deficits in one half of patients, and improvement of visual functioning in $1 / 3$ patients. Other retrospective data are in line with these findings [94]. Of note, long term side effects of brachytherapy seem to be rare in the paediatric subpopulation [96].

To summarize, studies on brachytherapy in pilocytic astrocytomas WHO grade I are rare and retrospective. Therefore, no clear conclusion can be drawn concerning indications for brachytherapy for these tumours. However, stereotactic iodine-125 brachytherapy has been shown to be a safe, minimally invasive, and an effective 
Table 3 Results concerning survival achieved with permanent and temporary iodine-125 brachytherapy in patients with various brain tumours.

\begin{tabular}{|c|c|c|c|c|c|c|c|c|c|c|c|c|c|c|c|}
\hline Study & $\begin{array}{l}\text { Number } \\
\text { of } \\
\text { patients } \\
\& \\
\text { type of } \\
\text { iodine- } \\
125 \text { seeds }\end{array}$ & $\begin{array}{l}\text { Dose, dose } \\
\text { rate, activity }\end{array}$ & $\begin{array}{l}\text { Diagnoses (number } \\
\text { of patients) \& } \\
\text { additional } \\
\text { percutaneous } \\
\text { radiotherapy (pRT) }\end{array}$ & $\begin{array}{l}6 \\
\text { months- } \\
\text { survival } \\
\text { rate }\end{array}$ & $\begin{array}{l}9 \\
\text { months- } \\
\text { survival } \\
\text { rate }\end{array}$ & $\begin{array}{l}12 \\
\text { months- } \\
\text { survival } \\
\text { rate }\end{array}$ & $\begin{array}{l}18 \\
\text { months- } \\
\text { survival } \\
\text { rate }\end{array}$ & $\begin{array}{l}2 \text { year- } \\
\text { survival } \\
\text { rate }\end{array}$ & $\begin{array}{l}3 \text { year- } \\
\text { survival } \\
\text { rate }\end{array}$ & $\begin{array}{l}4 \text { year- } \\
\text { survival } \\
\text { rate }\end{array}$ & $\begin{array}{l}5 \text { year- } \\
\text { survival } \\
\text { rate }\end{array}$ & $\begin{array}{l}8 \text { year- } \\
\text { survival } \\
\text { rate }\end{array}$ & $\begin{array}{l}10 \text { year- } \\
\text { survival } \\
\text { rate }\end{array}$ & $\begin{array}{l}15 \text { year- } \\
\text { survival } \\
\text { rate }\end{array}$ & $\begin{array}{l}\text { Median } \\
\text { survival }\end{array}$ \\
\hline \multirow[t]{6}{*}{$\begin{array}{l}\text { Mundinger } \\
1980[4]\end{array}$} & $\begin{array}{l}221 \\
\text { iodine-125 } \\
\text { \& iridium- } \\
192\end{array}$ & & Astrocytomas I (55) & & & & & & $72 \%$ & & $52 \%$ & & & & \\
\hline & & & Astrocytomas II (76) & & & & & & $69 \%$ & & $44 \%$ & & & & \\
\hline & & & Astrocytomas III (34) & & & & & & $66 \%$ & & $47 \%$ & & & & \\
\hline & & & $\begin{array}{l}\text { Oligodendrogliomas II/ } \\
\text { III (26) }\end{array}$ & & & & & & $65 \%$ & & $27 \%$ & & & & \\
\hline & & & Glioblastomas (17) & & & & & & & & & & & & \\
\hline & & & Germinomas (13) & & & & & $19 \%$ & $30 \%$ & & & & & & \\
\hline $\begin{array}{l}\text { Leibel } \\
1984 \text { [194] }\end{array}$ & $\begin{array}{l}43 \\
\text { temporary }\end{array}$ & 80-120Gy & $\begin{array}{l}\text { Recurrent primary } \\
\text { tumours } \\
\text { preirradiated }\end{array}$ & & & & & & & & & & & & $\begin{array}{l}>18 \\
\text { months }\end{array}$ \\
\hline $\begin{array}{l}\text { Wara } \\
1985 \text { [189] }\end{array}$ & temporary & & $\begin{array}{l}\text { Recurrent malignant } \\
\text { gliomas }\end{array}$ & & & & & & & & & & & & $\begin{array}{l}18 \\
\text { months }\end{array}$ \\
\hline $\begin{array}{l}\text { Leibel } \\
1985 \text { [195] }\end{array}$ & $\begin{array}{l}43 \\
\text { temporary }\end{array}$ & 80-120Gy & $\begin{array}{l}\text { Recurrent primary } \\
\text { tumours } \\
\text { preirradiated }\end{array}$ & & & & & & & & & & & & $\begin{array}{l}18 \\
\text { months }\end{array}$ \\
\hline \multirow[t]{3}{*}{$\begin{array}{l}\text { Leibel } \\
1986 \text { [111] }\end{array}$} & $\begin{array}{l}77 \\
\text { temporary }\end{array}$ & 50-120Gy & Recurrent tumours: & & & & & & & & & & & & \\
\hline & & & Astrocytomas III (42) & & & & & $49 \%$ & & & & & & & $\begin{array}{l}22 \\
\text { months }\end{array}$ \\
\hline & & & $\begin{array}{l}\text { Glioblastomas (35) } \\
\text { all preirradiated }\end{array}$ & & & & & $26 \%$ & & & & & & & $\begin{array}{l}17 \\
\text { months }\end{array}$ \\
\hline \multirow[t]{3}{*}{$\begin{array}{l}\text { Gutin } \\
1987 \text { [110] }\end{array}$} & $\begin{array}{l}41 \\
\text { temporary }\end{array}$ & 57.4-120Gy & Recurrent tumours: & & & & & & & & & & & & \\
\hline & & 25-100cGy/h & Astrocytomas III (23) & & & & & & & & & & & & $\begin{array}{l}35 \\
\text { months }\end{array}$ \\
\hline & & $30-40 \mathrm{mCi}$ & $\begin{array}{l}\text { Glioblastomas (18) } \\
\text { preirradiated (44- } \\
\text { 70.5Gy) }\end{array}$ & & & & & & & & & & & & $\begin{array}{l}8 \\
\text { months }\end{array}$ \\
\hline \multirow[t]{4}{*}{$\begin{array}{l}\text { Wright } \\
1987 \text { [47] }\end{array}$} & $\begin{array}{l}14 \\
\text { temporary }\end{array}$ & 100-280Gy & New tumours (12) & & & & & & & & & & & & $\begin{array}{l}2-32 \\
\text { months }\end{array}$ \\
\hline & $\begin{array}{l}2 \\
\text { permanent }\end{array}$ & $\begin{array}{l}\text { 15-30cGy/h } \\
6-144 \mathrm{mCi}\end{array}$ & Additional pRT (45Gy) & & & & & & & & & & & & \\
\hline & & & $\begin{array}{l}\text { Recurrent tumours (4) } \\
\text { Preirradiated }\end{array}$ & & & & & & & & & & & & \\
\hline & & & Glioblastomas (12) & & & & & & & & & & & & \\
\hline
\end{tabular}


Table 3 Results concerning survival achieved with permanent and temporary iodine-125 brachytherapy in patients with various brain tumours. (Continued)

\begin{tabular}{|c|c|c|c|c|c|c|c|c|}
\hline \multirow{6}{*}{$\begin{array}{l}\text { Willis } \\
1988 \text { [46] }\end{array}$} & 17 & New: & New glioblastomas (5) & $100 \%$ & $75 \%$ & $25 \%$ & & \\
\hline & & 60Gy & $\begin{array}{l}\text { Additional pRT } \\
\text { (50.4Gy) }\end{array}$ & & & & & \\
\hline & & $\begin{array}{l}35-64 c G y / h \\
34-239 m C i\end{array}$ & & & & & & \\
\hline & & Recurrent: & Recurrent tumours (12) 93\% & $60 \%$ & $50 \%$ & $38 \%$ & & \\
\hline & & 80Gy & Astrocytomas III (8) & & & & & \\
\hline & & $\begin{array}{l}30-65 \mathrm{cGy} / \mathrm{h} \\
122-320 \mathrm{mCi}\end{array}$ & $\begin{array}{l}\text { Glioblastomas (4) } \\
\text { preirradiated (50.4- } \\
66 \mathrm{~Gy})\end{array}$ & & & & & \\
\hline \multirow[t]{4}{*}{$\begin{array}{l}\text { Leibel } \\
1988 \text { [44] }\end{array}$} & $\begin{array}{l}95 \\
\text { temporary }\end{array}$ & 50-120Gy & & & & & & \\
\hline & & & Recurrent tumours: & & $55 \%$ & $40 \%$ & & $\begin{array}{l}20 \\
\text { months }\end{array}$ \\
\hline & & & Astrocytomas III (50) & & $30 \%$ & $15 \%$ & & $\begin{array}{l}12 \\
\text { months }\end{array}$ \\
\hline & & & $\begin{array}{l}\text { Glioblastomas (45) } \\
\text { preirradiated }\end{array}$ & & & & & \\
\hline $\begin{array}{l}\text { Prados } \\
1989 \text { [158] }\end{array}$ & $\begin{array}{l}14 \\
\text { temporary }\end{array}$ & $\begin{array}{l}50 \mathrm{cGy} / \mathrm{h} \\
10-40 \mathrm{mCi}\end{array}$ & $\begin{array}{l}\text { Metastases } \\
\text { preirradiated }\end{array}$ & & & & & $\begin{array}{l}18 \\
\text { months }\end{array}$ \\
\hline \multirow[t]{4}{*}{$\begin{array}{l}\text { Etou } \\
1989 \text { [93] }\end{array}$} & $\begin{array}{l}56 \\
\text { temporary } \\
\& \\
\text { permanent }\end{array}$ & 90-100Gy & Paediatric tumours: & & & & & \\
\hline & & & Astrocytomas I (36) & $86 \%$ & & $58 \%$ & $22 \%$ & \\
\hline & & & Astrocytomas II (14) & $79 \%$ & & $36 \%$ & $21 \%$ & \\
\hline & & & Astrocytomas III (6) & $33 \%$ & & $17 \%$ & $17 \%$ & \\
\hline \multirow[t]{3}{*}{$\begin{array}{l}\text { Malkin } \\
1989[127]\end{array}$} & $\begin{array}{l}21 \\
\text { temporary }\end{array}$ & 60Gy & Astrocytomas III (2) & & & & & \\
\hline & & 36cGy/h & Glioblastomas (19) & & & & & $\begin{array}{l}6 \\
\text { months }\end{array}$ \\
\hline & & $79-495 \mathrm{mCi}$ & $\begin{array}{l}\text { Recurrent tumours } \\
\text { (10): } \\
\text { Preirradiated }\end{array}$ & & & & & \\
\hline \multirow[t]{5}{*}{$\begin{array}{l}\text { Yakar } \\
1989 \text { [159] }\end{array}$} & $\begin{array}{l}50 \\
\text { temporary }\end{array}$ & 55-60Gy & New tumours: & & & & & $\begin{array}{l}11 \\
\text { months } \\
\text { (mean) }\end{array}$ \\
\hline & & 40cGy/h & Astrocytomas III (3) & & & & & \\
\hline & & 4-299mCi & Glioblastomas (17) & & & & & \\
\hline & & & $\begin{array}{l}\text { Additional pRT (50- } \\
\text { 60Gy) }\end{array}$ & & & & & \\
\hline & & & Recurrent tumours: & & & & & $\begin{array}{l}13 \\
\text { months }\end{array}$ \\
\hline
\end{tabular}


Table 3 Results concerning survival achieved with permanent and temporary iodine-125 brachytherapy in patients with various brain tumours. (Continued)

\begin{tabular}{|c|c|c|c|c|c|c|}
\hline & & & Astrocytomas III (5) & & & \\
\hline & & & Glioblastomas (12) & & & \\
\hline & & & Preirradiated & & & \\
\hline & & & Metastases (13) & & & $\begin{array}{l}8 \\
\text { months } \\
\text { (mean) }\end{array}$ \\
\hline \multirow[t]{3}{*}{$\begin{array}{l}\text { Leibel } \\
1989 \text { [45] }\end{array}$} & $\begin{array}{l}95 \\
\text { temporary }\end{array}$ & 52.7-150Gy & Recurrent tumours: & & & \\
\hline & & 20-90cGy/h & Astrocytomas III (50) & $46 \%$ & $28 \%$ & $\begin{array}{l}19 \\
\text { months }\end{array}$ \\
\hline & & $27-372 \mathrm{mCi}$ & $\begin{array}{l}\text { Glioblastomas (45) } \\
\text { preirradiated (40-72Gy) }\end{array}$ & $22 \%$ & $8 \%$ & $\begin{array}{l}12 \\
\text { months }\end{array}$ \\
\hline \multirow[t]{7}{*}{$\begin{array}{l}\text { Larson } \\
1990[81]\end{array}$} & $\begin{array}{l}128 \\
\text { temporary }\end{array}$ & 46-120Gy & New tumours: & & & \\
\hline & & 25-100cGy/h & Astrocytomas III (20) & & & $\begin{array}{l}51 \\
\text { months }\end{array}$ \\
\hline & & & Glioblastomas (13) & & & $\begin{array}{l}22 \\
\text { months }\end{array}$ \\
\hline & & & Additional pRT (60Gy) & & & \\
\hline & & & Recurrent tumours: & & & \\
\hline & & & Astrocytomas III (50) & $45 \%$ & $28 \%$ & $\begin{array}{l}19 \\
\text { months }\end{array}$ \\
\hline & & & $\begin{array}{l}\text { Glioblastomas (45) } \\
\text { Preirradiated }\end{array}$ & $22 \%$ & $8 \%$ & $\begin{array}{l}12 \\
\text { months }\end{array}$ \\
\hline \multirow[t]{7}{*}{$\begin{array}{l}\text { Bernstein } \\
1990 \text { [107] }\end{array}$} & $\begin{array}{l}46 \\
\text { temporary }\end{array}$ & New: & New tumours: & & & \\
\hline & & 60Gy & Astrocytomas III (23) & & & $\begin{array}{l}14 \\
\text { months }\end{array}$ \\
\hline & & & Additional pRT (50Gy) & & & \\
\hline & & Recurrent: & Recurrent tumours: & & & \\
\hline & & 70Gy & Astrocytomas III (18) & & & $\begin{array}{l}10 \\
\text { months }\end{array}$ \\
\hline & & $\begin{array}{l}21.8-125 \mathrm{cGy} / \\
\text { h } 93-599 \mathrm{mCi}\end{array}$ & Metastases (3) & & & \\
\hline & & & $\begin{array}{l}\text { Radiation-induced } \\
\text { tumours (2) } \\
\text { preirradiated }\end{array}$ & & & \\
\hline \multirow[t]{2}{*}{$\begin{array}{l}\text { Loeffler } \\
1990 \text { [134] }\end{array}$} & $\begin{array}{l}35 \\
\text { temporary }\end{array}$ & 38-55Gy & Glioblastomas (35) & $87 \%$ & $57 \%$ & \\
\hline & & $\begin{array}{l}30-60 \mathrm{cGy} / \mathrm{h} \\
20-50 \mathrm{mCi}\end{array}$ & $\begin{array}{l}\text { Additional pRT } \\
\text { (59.4Gy) }\end{array}$ & & & \\
\hline $\begin{array}{l}\text { Gutin } \\
1990 \text { [109] }\end{array}$ & $\begin{array}{l}55 \\
\text { temporary }\end{array}$ & 50Gy & Astrocytomas III (25) & & & $\begin{array}{l}36 \\
\text { months }\end{array}$ \\
\hline
\end{tabular}


Table 3 Results concerning survival achieved with permanent and temporary iodine-125 brachytherapy in patients with various brain tumours. (Continued)

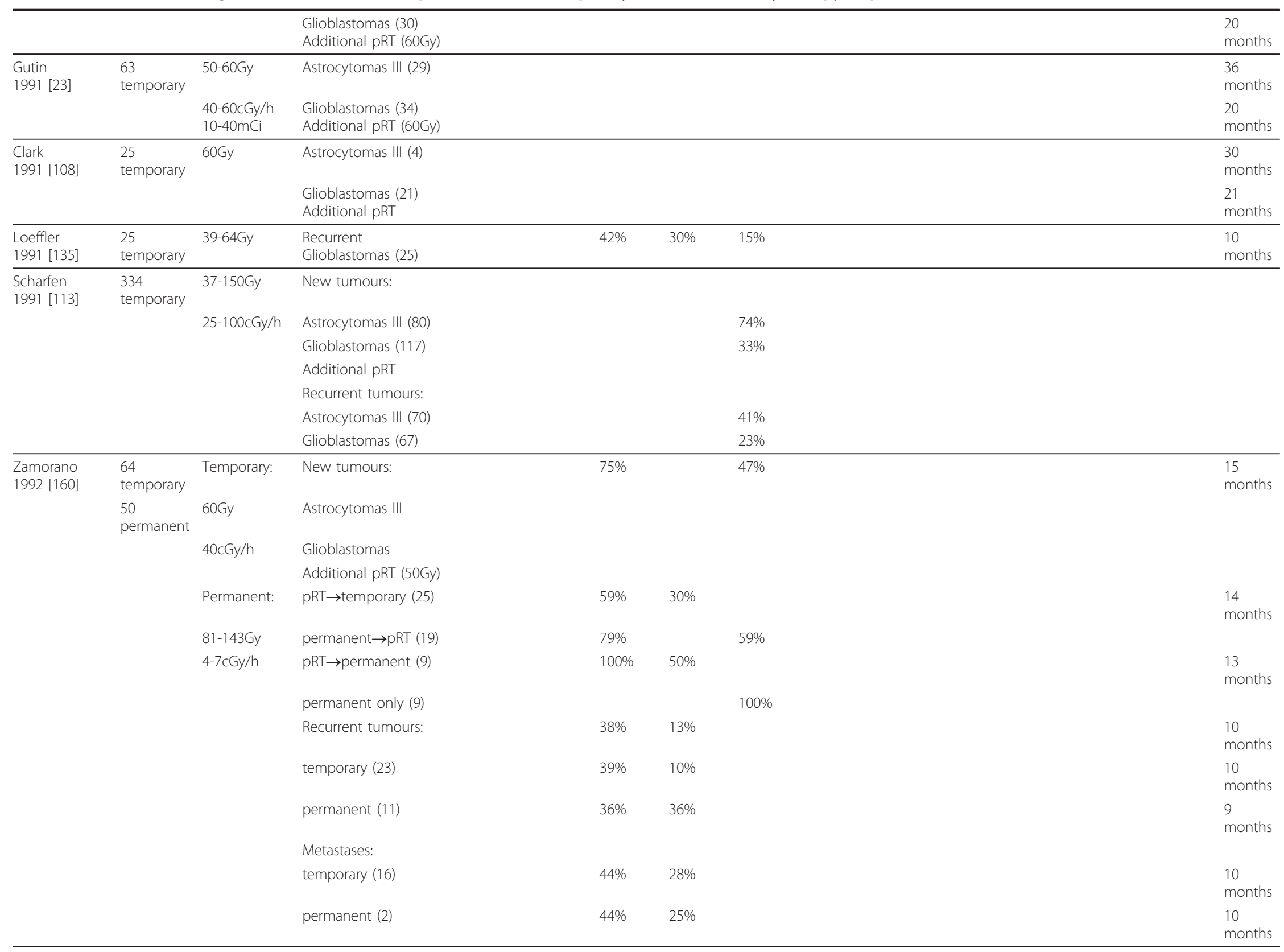


Table 3 Results concerning survival achieved with permanent and temporary iodine-125 brachytherapy in patients with various brain tumours. (Continued)

\begin{tabular}{|c|c|c|c|c|c|}
\hline \multirow[t]{9}{*}{$\begin{array}{l}\text { Zamorano } \\
1992 \text { [161] }\end{array}$} & $\begin{array}{l}50 \\
\text { temporary }\end{array}$ & 55-60Gy & New tumours: & & $\begin{array}{l}11 \\
\text { months } \\
\text { (mean) }\end{array}$ \\
\hline & & 40cGy/h & Astrocytomas III (3) & & \\
\hline & & 4-299mCi & Glioblastomas (17) & & \\
\hline & & & $\begin{array}{l}\text { Additional pRT (50- } \\
60 \mathrm{~Gy} \text { ) }\end{array}$ & & $\begin{array}{l}13 \\
\text { months }\end{array}$ \\
\hline & & & Recurrent tumours: & & \\
\hline & & & Astrocytomas III (5) & & \\
\hline & & & Glioblastomas (12) & & \\
\hline & & & Preirradiated & & \\
\hline & & & Metastases (13) & & $\begin{array}{l}8 \\
\text { months } \\
\text { (mean) }\end{array}$ \\
\hline \multirow[t]{12}{*}{$\begin{array}{l}\text { Sneed } \\
1992 \text { [145] }\end{array}$} & $\begin{array}{l}206 \\
\text { temporary }\end{array}$ & New: & New tumours: & & \\
\hline & & 42-76Gy & Astrocytomas III (29) & & $\begin{array}{l}36 \\
\text { months }\end{array}$ \\
\hline & & & Glioblastomas (34) & & $\begin{array}{l}20 \\
\text { months }\end{array}$ \\
\hline & & & Additional pRT (60Gy) & & \\
\hline & & Recurrent: & Recurrent tumours: & & \\
\hline & & 53-150Gy & Astrocytomas III (50) & & $\begin{array}{l}19 \\
\text { months }\end{array}$ \\
\hline & & 20-90cGy/h & Glioblastomas (45) & & $\begin{array}{l}12 \\
\text { months }\end{array}$ \\
\hline & & & Preirradiated & & \\
\hline & & Hyperthermia: & Recurrent tumours: & & \\
\hline & & 33-63Gy & Astrocytomas III (16) & $65 \%$ & \\
\hline & & & Glioblastomas (25) & $45 \%$ & $\begin{array}{l}11 \\
\text { months }\end{array}$ \\
\hline & & & $\begin{array}{l}\text { Metastases (7) } \\
\text { Plus hyperthermia }\end{array}$ & & $\begin{array}{l}10 \\
\text { months }\end{array}$ \\
\hline \multirow[t]{5}{*}{$\begin{array}{l}\text { Yakar } \\
1992 \text { [115] }\end{array}$} & $\begin{array}{l}62 \\
\text { temporary }\end{array}$ & Temporary: & New tumours: & $40 \%$ & $\begin{array}{l}14 \\
\text { months }\end{array}$ \\
\hline & & 60Gy & Astrocytomas III (7) & $43 \%$ & $\begin{array}{l}13 \\
\text { months }\end{array}$ \\
\hline & & 40cGy/h & Glioblastomas (18) & $39 \%$ & $\begin{array}{l}15 \\
\text { months }\end{array}$ \\
\hline & & & Additional pRT & & \\
\hline & & & Recurrent tumours: & $23 \%$ & $\begin{array}{l}11 \\
\text { months }\end{array}$ \\
\hline
\end{tabular}


Table 3 Results concerning survival achieved with permanent and temporary iodine-125 brachytherapy in patients with various brain tumours. (Continued)

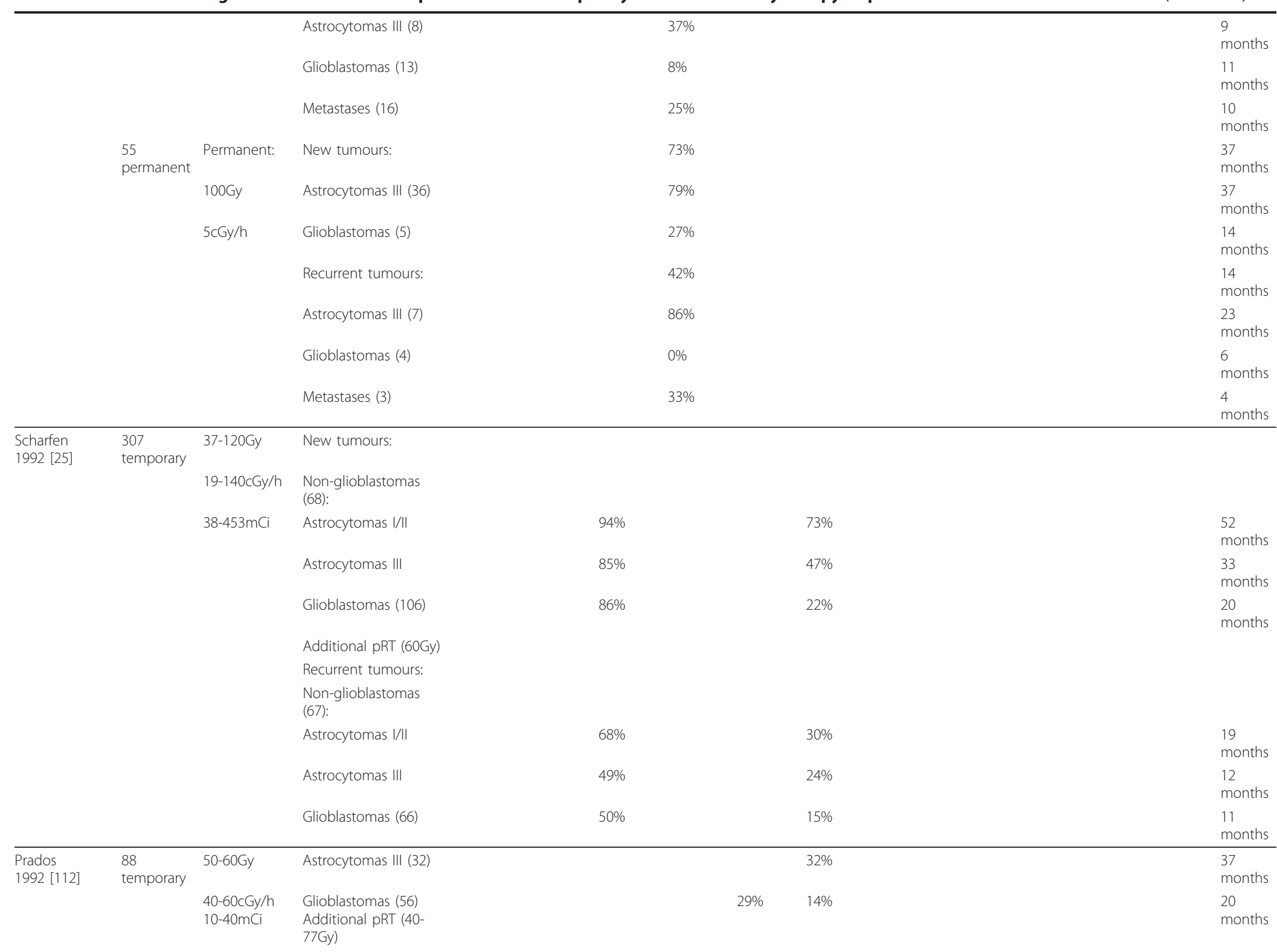


Table 3 Results concerning survival achieved with permanent and temporary iodine-125 brachytherapy in patients with various brain tumours. (Continued)

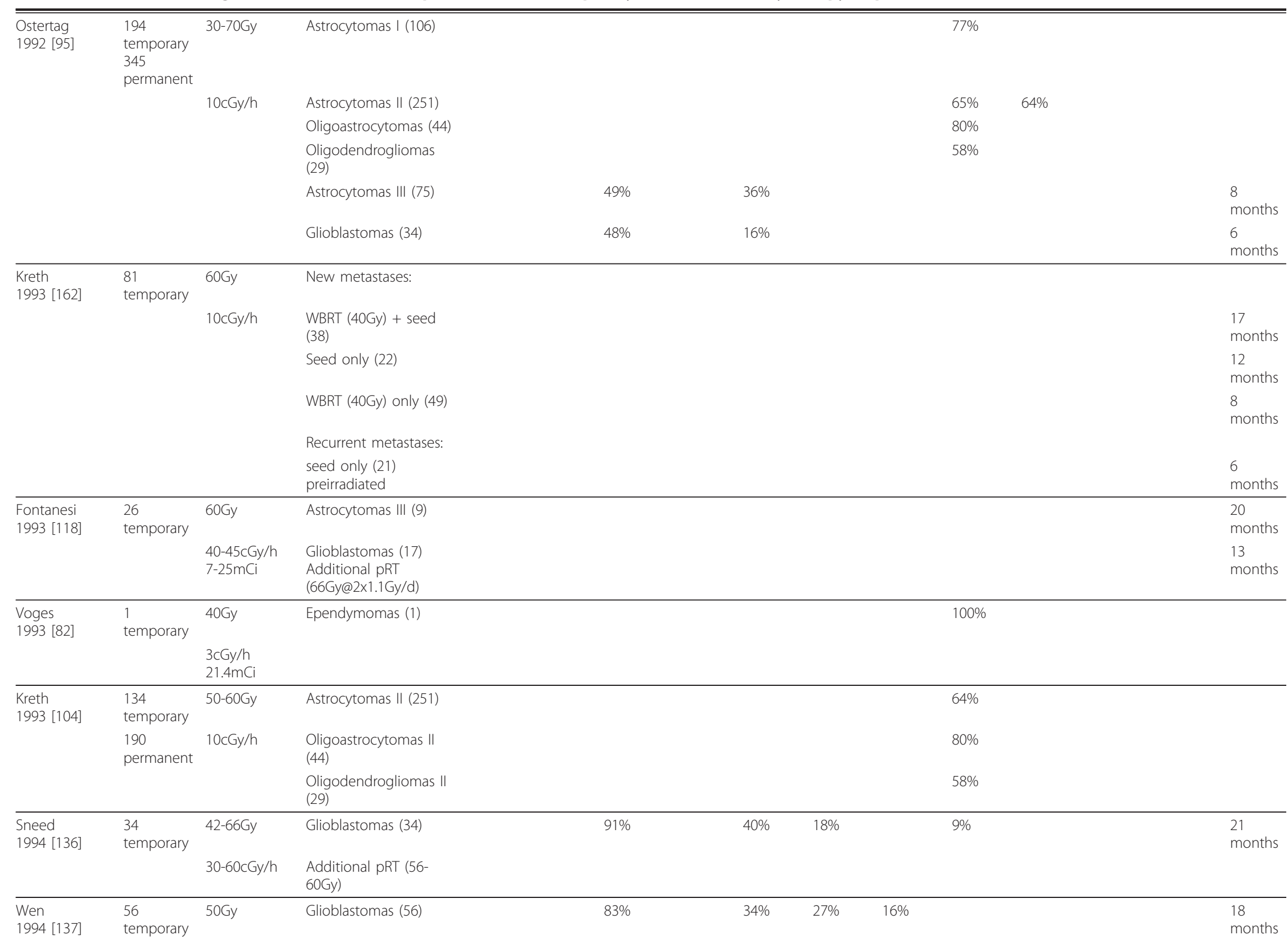


Table 3 Results concerning survival achieved with permanent and temporary iodine-125 brachytherapy in patients with various brain tumours. (Continued)

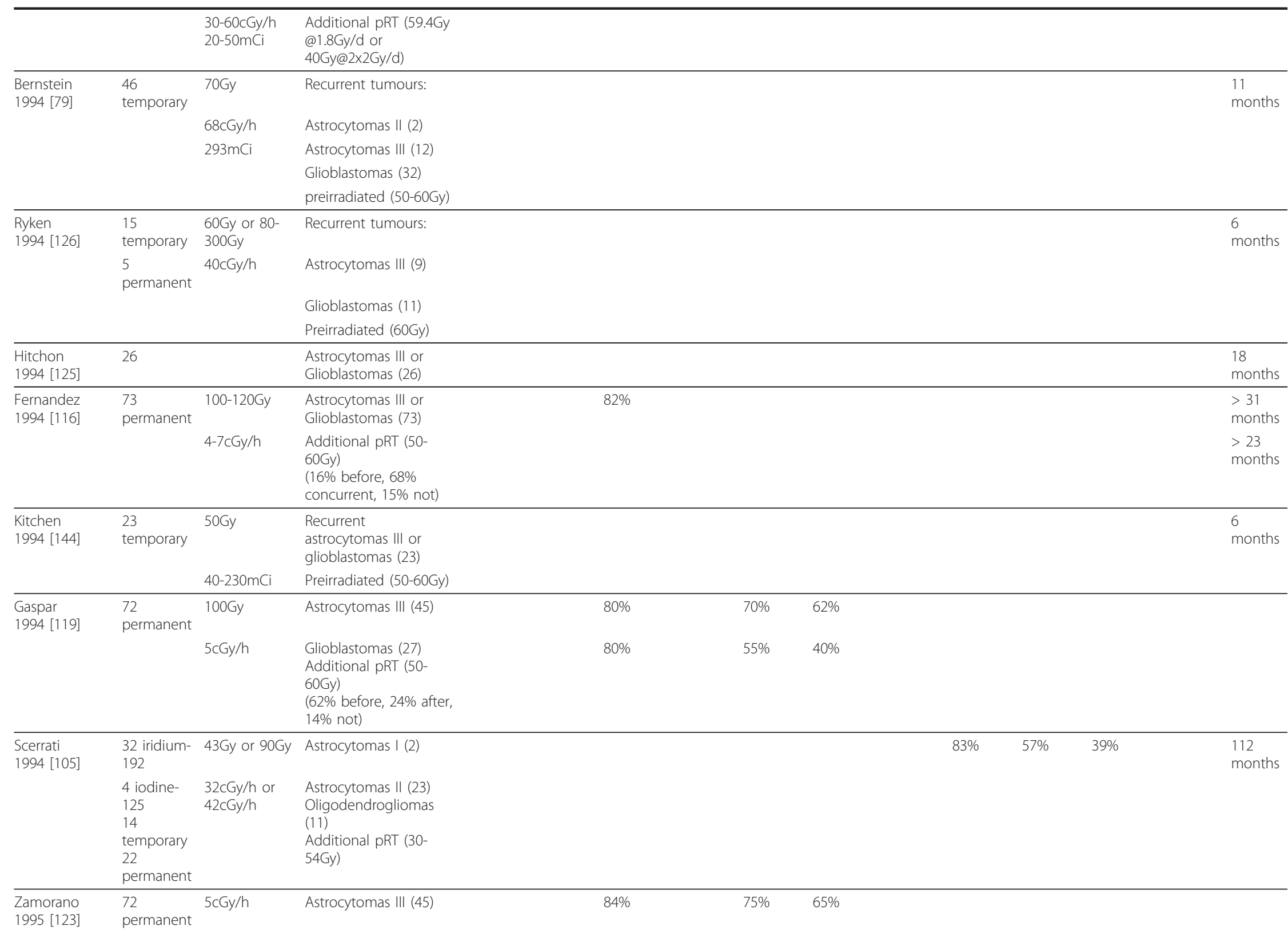


Table 3 Results concerning survival achieved with permanent and temporary iodine-125 brachytherapy in patients with various brain tumours. (Continued)

\begin{tabular}{|c|c|c|c|c|c|c|c|c|c|}
\hline & & & $\begin{array}{l}\text { Glioblastomas ( } 27) \\
\text { Additional pRT ( } 50- \\
60 G y) \\
\text { (24\% before, } 63 \% \text { after, } \\
14 \% \text { not) }\end{array}$ & $80 \%$ & $53 \%$ & & & & $\begin{array}{l}26 \\
\text { months }\end{array}$ \\
\hline \multirow{6}{*}{$\begin{array}{l}\text { Chamberlain } \\
1995 \text { [185] }\end{array}$} & & 45-51Gy & Recurrent tumours: & & & & & & \\
\hline & $\begin{array}{l}16 \\
\text { temporary }\end{array}$ & $50 c G y / h$ & Astrocytomas III (3) & & & & & & $\begin{array}{l}9 \\
\text { months }\end{array}$ \\
\hline & & & $\begin{array}{l}\text { Oligodendrogliomas III } \\
\text { (1) }\end{array}$ & & & & & & \\
\hline & & & Glioblastomas (11) & & & & & & \\
\hline & & & Ependymomas (1) & & & & & & \\
\hline & & & Preirradiated (54-60Gy) & & & & & & \\
\hline \multirow[t]{5}{*}{$\begin{array}{l}\text { Kreth } \\
1995 \text { [68] }\end{array}$} & $\begin{array}{l}194 \\
\text { temporary } \\
261 \\
\text { permanent }\end{array}$ & 60-100Gy & Astrocytomas I (97) & & & & $85 \%$ & $83 \%$ & \\
\hline & & $<10 c G y / h$ & Astrocytomas II (250) & & & & $61 \%$ & $51 \%$ & \\
\hline & & & Oligoastrocytomas (60) & & & & $49 \%$ & & $\begin{array}{l}58 \\
\text { months }\end{array}$ \\
\hline & & & $\begin{array}{l}\text { Oligodendrogliomas } \\
\text { (27) }\end{array}$ & & & & $50 \%$ & & $\begin{array}{l}61 \\
\text { months }\end{array}$ \\
\hline & & & $\begin{array}{l}\text { Gemistocytic gliomas } \\
(21)\end{array}$ & & & & $32 \%$ & & $\begin{array}{l}37 \\
\text { months }\end{array}$ \\
\hline \multirow[t]{3}{*}{$\begin{array}{l}\text { Fernandez } \\
1995[117]\end{array}$} & $\begin{array}{l}58 \\
\text { permanent }\end{array}$ & 102Gy & Astrocytomas III (40) & & & & & & $\begin{array}{l}>31 \\
\text { months }\end{array}$ \\
\hline & & 4-7cGy/h & Glioblastomas (18) & & & & & & $\begin{array}{l}>23 \\
\text { months }\end{array}$ \\
\hline & & $0.1-32 \mathrm{mCi}$ & $\begin{array}{l}\text { Additional pRT (50- } \\
60 \mathrm{~Gy}) \\
(17 \% \text { before, } 66 \% \text { after, } \\
17 \% \text { not) }\end{array}$ & & & & & & \\
\hline \multirow[t]{2}{*}{$\begin{array}{l}\text { Sneed } \\
1995 \text { [141] }\end{array}$} & $\begin{array}{l}159 \\
\text { temporary }\end{array}$ & 36-67Gy & Glioblastomas (159) & $85 \%$ & $36 \%$ & $20 \%$ & & & $\begin{array}{l}19 \\
\text { months }\end{array}$ \\
\hline & & 30-70cGy/h & $\begin{array}{l}\text { Additional pRT (40- } \\
\text { 77Gy) }\end{array}$ & & & & & & \\
\hline \multirow[t]{4}{*}{$\begin{array}{l}\text { Ostertag } \\
1995[150]\end{array}$} & $\begin{array}{l}93 \\
\text { temporary }\end{array}$ & $\begin{array}{l}\text { 60-100Gy } \\
10 \mathrm{cGy} / \mathrm{h}\end{array}$ & New metastases: & & & & & & \\
\hline & & & $\begin{array}{l}\text { Seed + pRT (40Gy) } \\
\text { (38) }\end{array}$ & & & & & & $\begin{array}{l}17 \\
\text { months }\end{array}$ \\
\hline & & & seed only (34) & & & & & & $\begin{array}{l}15 \\
\text { months }\end{array}$ \\
\hline & & & $\begin{array}{l}\text { Recurrent Metastases: } \\
\text { Preirradiated ( } 21)\end{array}$ & & & & & & $\begin{array}{l}6 \\
\text { months }\end{array}$ \\
\hline
\end{tabular}


Table 3 Results concerning survival achieved with permanent and temporary iodine-125 brachytherapy in patients with various brain tumours. (Continued)

\begin{tabular}{|c|c|c|c|c|c|c|c|c|c|}
\hline \multirow[t]{3}{*}{$\begin{array}{l}\text { Halligan } \\
1996[128]\end{array}$} & $\begin{array}{l}22 \\
\text { permanent }\end{array}$ & 150-300Gy & Recurrent tumours: & $57 \%$ & & & & & $\begin{array}{l}15 \\
\text { months }\end{array}$ \\
\hline & & 11cGy/h & Astrocytomas III (4) & & & & & & \\
\hline & & $39 \mathrm{mCi}$ & $\begin{array}{l}\text { Glioblastomas (18) } \\
\text { Preirradiated (54-65Gy) }\end{array}$ & $59 \%$ & & & & & $\begin{array}{l}15 \\
\text { months }\end{array}$ \\
\hline \multirow[t]{3}{*}{$\begin{array}{l}\text { Kreth } \\
1997 \text { [99] }\end{array}$} & $\begin{array}{l}197 \\
\text { temporary } \\
\& \\
\text { permanent }\end{array}$ & 45-100Gy or & Astrocytomas II (153) & & & & $60 \%$ & & \\
\hline & & 50-120Gy & $\begin{array}{l}\text { Oligoastrocytomas II } \\
\text { (44) }\end{array}$ & & & & & & \\
\hline & & 3-18cGy/h & Additional pRT (47) & & & & & & $\begin{array}{l}17 \\
\text { months }\end{array}$ \\
\hline \multirow{2}{*}{$\begin{array}{l}\text { Sneed } \\
1997 \text { [12] }\end{array}$} & permanent & 60-100Gy & Astrocytomas I & & & & $85 \%$ & $83 \%$ & \\
\hline & & 5-10cGy/h & Astrocytomas II & & & & $61 \%$ & $51 \%$ & \\
\hline \multirow[t]{2}{*}{$\begin{array}{l}\text { Sneed } \\
1997 \text { [12] }\end{array}$} & temporary & 60Gy & $\begin{array}{l}\text { New high-grade } \\
\text { gliomas }\end{array}$ & & & & & & $\begin{array}{l}\text { 18-19 } \\
\text { months }\end{array}$ \\
\hline & & $40-60 c G y / h$ & $\begin{array}{l}\text { Recurrent high-grade } \\
\text { gliomas }\end{array}$ & & & & & & $\begin{array}{l}12-13 \\
\text { months }\end{array}$ \\
\hline \multirow[t]{2}{*}{$\begin{array}{l}\text { Schulder } \\
1997 \text { [151] }\end{array}$} & $\begin{array}{l}13 \\
\text { permanent }\end{array}$ & 43-132Gy & Metastases (13) & & & & & & $\begin{array}{l}8 \\
\text { months }\end{array}$ \\
\hline & & $0.26-0.79 \mathrm{mCi}$ & $\begin{array}{l}\text { Additional pRT (30- } \\
\text { 75Gy) }\end{array}$ & & & & & & \\
\hline $\begin{array}{l}\text { Chamberlain } \\
1997 \text { [138] }\end{array}$ & $\begin{array}{l}15 \\
\text { temporary }\end{array}$ & $\begin{array}{l}\text { 50Gy } \\
\text { 50cGy/h }\end{array}$ & $\begin{array}{l}\text { Recurrent } \\
\text { glioblastomas (15) }\end{array}$ & & & & & & $\begin{array}{l}10 \\
\text { months }\end{array}$ \\
\hline \multirow[t]{3}{*}{$\begin{array}{l}\text { Laperriere } \\
1998[24]\end{array}$} & $\begin{array}{l}63 \\
\text { temporary }\end{array}$ & 57-68Gy & $\begin{array}{l}\text { Malignant } \\
\text { astrocytomas (140) }\end{array}$ & & & & & & \\
\hline & & $21-125 \mathrm{cGy} / \mathrm{h}$ & $\begin{array}{l}\text { Additional pRT (50Gy) } \\
\text { (63) }\end{array}$ & & & & & & $\begin{array}{l}16 \\
\text { months }\end{array}$ \\
\hline & & & only pRT (50Gy) (69) & & & & & & $\begin{array}{l}13 \\
\text { months }\end{array}$ \\
\hline \multirow[t]{4}{*}{$\begin{array}{l}\text { Gaspar } \\
1999[53]\end{array}$} & $\begin{array}{l}59 \\
\text { permanent }\end{array}$ & 100Gy & Recurrent tumours: & & & & & & \\
\hline & & $5 c G y / h$ & Astrocytomas III (22) & $76 \%$ & $55 \%$ & $32 \%$ & & & $\begin{array}{l}16 \\
\text { months }\end{array}$ \\
\hline & & & Glioblastomas (37) & $44 \%$ & $13 \%$ & & & & $\begin{array}{l}24 \\
\text { months }\end{array}$ \\
\hline & & & preirradiated (50-66Gy) & & & & & & $\begin{array}{l}11 \\
\text { months }\end{array}$ \\
\hline $\begin{array}{l}\text { Videtic } \\
\text { 1999, } 2001\end{array}$ & $\begin{array}{l}75 \\
\text { permanent }\end{array}$ & 100Gy & Astrocytomas III (22) & & $58 \%$ & & $40 \%$ & & $\begin{array}{l}40 \\
\text { months }\end{array}$ \\
\hline
\end{tabular}

permanent 
Table 3 Results concerning survival achieved with permanent and temporary iodine-125 brachytherapy in patients with various brain tumours. (Continued)

\begin{tabular}{|c|c|c|c|c|c|c|c|c|}
\hline & & $5 c G y / h$ & $\begin{array}{l}\text { Glioblastomas (53) } \\
\text { Additional pRT (50- } \\
\text { 60Gy) }\end{array}$ & & $40-42 \%$ & $18 \%$ & & $\begin{array}{l}\text { 16-17 } \\
\text { months }\end{array}$ \\
\hline \multirow[t]{2}{*}{$\begin{array}{l}\text { Suplica } \\
1999 \text { [142] }\end{array}$} & $\begin{array}{l}38 \\
\text { permanent }\end{array}$ & 100-500Gy & $\begin{array}{l}\text { Recurrent } \\
\text { glioblastomas (38) }\end{array}$ & & & & & $\begin{array}{l}12 \\
\text { months }\end{array}$ \\
\hline & & $0.67 \mathrm{mCi}$ & Preirradiated (59-72Gy) & & & & & \\
\hline $\begin{array}{l}\text { Patel } \\
2000 \text { [140] }\end{array}$ & $\begin{array}{l}40 \\
\text { permanent }\end{array}$ & $\begin{array}{l}120-160 \mathrm{~Gy} \\
0.58-0.86 \mathrm{mCi}\end{array}$ & $\begin{array}{l}\text { Recurrent } \\
\text { glioblastomas (40) } \\
\text { preirradiated (60Gy) }\end{array}$ & & & & & $\begin{array}{l}11 \\
\text { months }\end{array}$ \\
\hline $\begin{array}{l}\text { Koot } \\
2000 \text { [139] } \\
\text { comment } \\
{[196]}\end{array}$ & $\begin{array}{l}33 \\
\text { temporary } \\
12 \\
\text { permanent }\end{array}$ & $\begin{array}{l}50-80 G y \\
3-5 c G y / h \\
5-40 \mathrm{mCi}\end{array}$ & $\begin{array}{l}\text { Glioblastomas (45) } \\
\text { Additional pRT (10- } \\
\text { 30Gy) }\end{array}$ & & & & & $\begin{array}{l}13 \\
\text { months }\end{array}$ \\
\hline \multirow[t]{6}{*}{$\begin{array}{l}\text { Rostomily } \\
2001 \text { [197] }\end{array}$} & $\begin{array}{l}6 \\
\text { permanent }\end{array}$ & 160-218Gy & $\begin{array}{l}\text { Recurrent paediatric } \\
\text { tumours: }\end{array}$ & & & & & $\begin{array}{l}22 \\
\text { months }\end{array}$ \\
\hline & & $\begin{array}{l}10 \mathrm{cGy} / \mathrm{h} \\
23-76 \mathrm{mCi}\end{array}$ & $\begin{array}{l}\text { Primitive } \\
\text { neuroectodermal } \\
\text { tumours (2) }\end{array}$ & & & & & \\
\hline & & & Medulloblastomas (1) & & & & & \\
\hline & & & Ependymomas (1) & & & & & \\
\hline & & & $\begin{array}{l}\text { Xanthoastrocytomas } \\
\text { (1) }\end{array}$ & & & & & \\
\hline & & & $\begin{array}{l}\text { Glioblastomas (1) } \\
\text { preirradiated (3 } \\
\text { craniospinal, } 2 \text { WBRT) }\end{array}$ & & & & & \\
\hline $\begin{array}{l}\text { Keole } \\
2001 \text { [132] }\end{array}$ & $\begin{array}{l}26 \\
\text { permanent }\end{array}$ & 83-100Gy & $\begin{array}{l}\text { Glioblastomas (26) } \\
\text { Additional pRT (57- } \\
61 \text { Gy) }\end{array}$ & & & & & $\begin{array}{l}23 \\
\text { months }\end{array}$ \\
\hline \multirow[t]{3}{*}{$\begin{array}{l}\text { Huang } \\
2002 \text { [153] }\end{array}$} & $\begin{array}{l}28 \\
\text { permanent }\end{array}$ & 200-500Gy & Metastases: & & & & & $\begin{array}{l}11 \\
\text { months }\end{array}$ \\
\hline & & $15-69 \mathrm{mCi}$ & New (16) & & & & & $\begin{array}{l}14 \\
\text { months }\end{array}$ \\
\hline & & & Recurrent (12) & & & & & $\begin{array}{l}5 \\
\text { months }\end{array}$ \\
\hline \multirow[t]{2}{*}{$\begin{array}{l}\text { Zamorano } \\
2003[124]\end{array}$} & $\begin{array}{l}60 \\
\text { permanent }\end{array}$ & 100Gy & Astrocytomas III (57) & $87 \%$ & $60 \%$ & $50 \%$ & $46 \%$ & $\begin{array}{l}57 \\
\text { months }\end{array}$ \\
\hline & & 3-7cGy/h & $\begin{array}{l}\text { Oligodendrogliomas III } \\
\text { (3) } \\
\text { Additional pRT (50- } \\
60 \mathrm{~Gy}) \\
\text { (78\% before, 22\% not) }\end{array}$ & & & & & \\
\hline $\begin{array}{l}\text { Larson } \\
2004 \text { [133] }\end{array}$ & $\begin{array}{l}35 \\
\text { permanent }\end{array}$ & $\begin{array}{l}150-500 G y \\
7-24 c G y / h \\
56 \mathrm{mCi}\end{array}$ & $\begin{array}{l}\text { Recurrent } \\
\text { glioblastomas (35) } \\
\text { Preirradiated (59-72Gy) }\end{array}$ & & & & & $\begin{array}{l}12 \\
\text { months }\end{array}$ \\
\hline
\end{tabular}


Table 3 Results concerning survival achieved with permanent and temporary iodine-125 brachytherapy in patients with various brain tumours. (Continued)

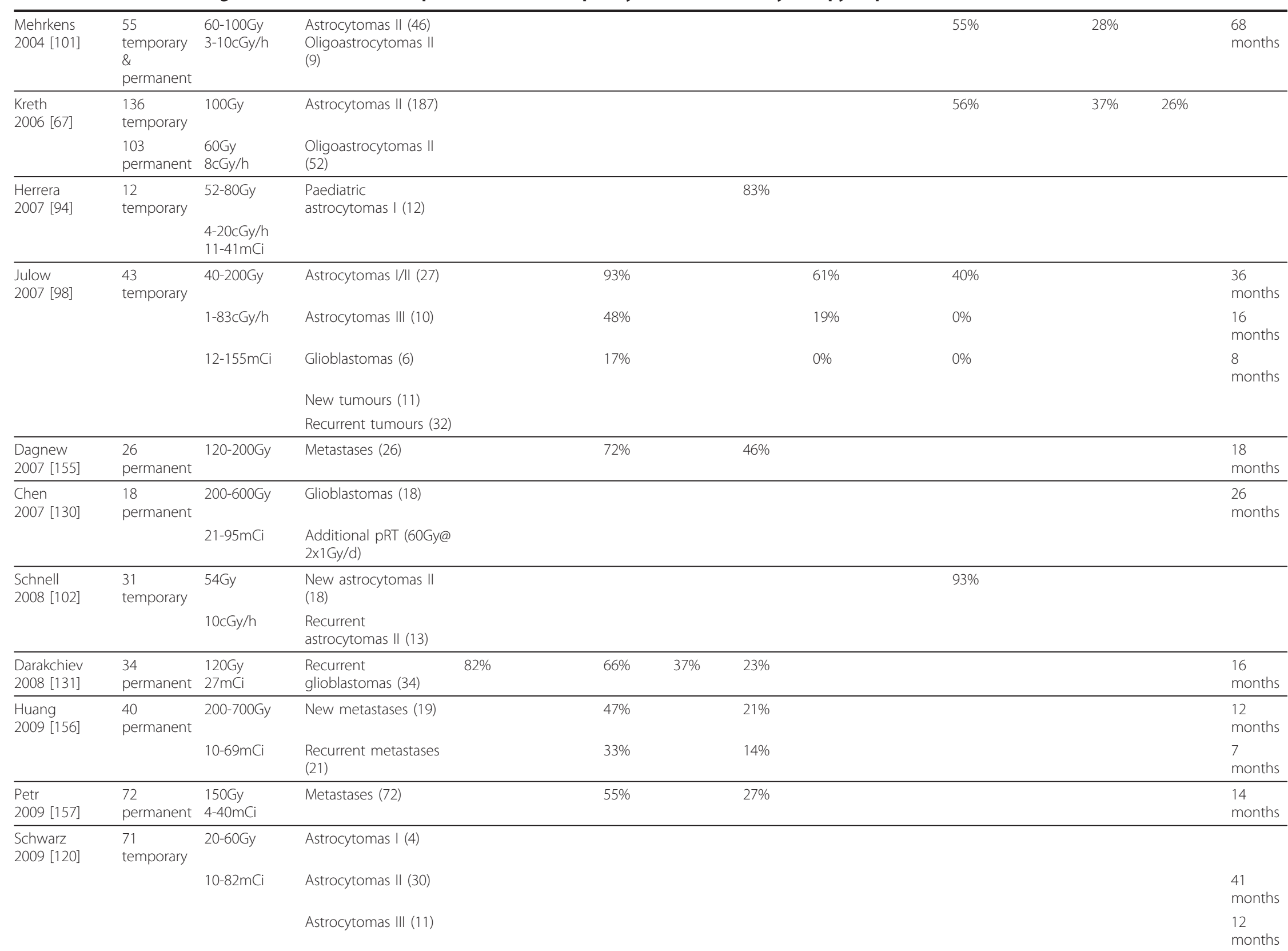


Table 3 Results concerning survival achieved with permanent and temporary iodine-125 brachytherapy in patients with various brain tumours. (Continued)

\begin{tabular}{|c|c|c|c|c|c|c|c|c|}
\hline & & & Glioblastomas (13) & & & & & 4 \\
\hline & & & Medulloblastomas (4) & & & & & \\
\hline & & & $\begin{array}{l}\text { Oligodendrogliomas } \\
\text { (3) }\end{array}$ & & & & & \\
\hline & & & Pinealis tumour (1) & & & & & \\
\hline & & & Metastases (5) & & & & & \\
\hline & & & Preirradiated (18-80Gy) & & & & & $\begin{array}{l}22 \\
\text { months }\end{array}$ \\
\hline $\begin{array}{l}\text { Ruge } 2011 \\
\text { [149] }\end{array}$ & $\begin{array}{l}77 \\
\text { temporary }\end{array}$ & $\begin{array}{l}50 G y \\
6 c G y / h\end{array}$ & Metastases (77) & & & & & $\begin{array}{l}8 \\
\text { months }\end{array}$ \\
\hline \multirow[t]{7}{*}{$\begin{array}{l}\text { Korinthenberg } \\
2011 \text { [198] }\end{array}$} & $\begin{array}{l}94 \\
\text { temporary }\end{array}$ & $60 \mathrm{~Gy}$ & Paediatric tumours: & & & $97 \%$ & $92 \%$ & \\
\hline & & 10cGy/h & Astrocytomas I (53) & & & & & \\
\hline & & $10 \mathrm{mCi}$ & Astrocytomas II (26) & & & & & \\
\hline & & & $\begin{array}{l}\text { Oligoastrocytomas II } \\
\text { (4) }\end{array}$ & & & & & \\
\hline & & & $\begin{array}{l}\text { Oligodendrogliomas II } \\
\text { (5) }\end{array}$ & & & & & \\
\hline & & & Ependymoma II (1) & & & & & \\
\hline & & & Not classified (5) & & & & & \\
\hline \multirow[t]{2}{*}{$\begin{array}{l}\text { El Majdoub } \\
2011 \text { [183] }\end{array}$} & $\begin{array}{l}11 \\
\text { temporary }\end{array}$ & 32-50Gy & $\begin{array}{l}\text { Recurrent } \\
\text { Medulloblastomas (12) }\end{array}$ & $67 \%$ & $33 \%$ & & & $\begin{array}{l}18 \\
\text { months }\end{array}$ \\
\hline & $\begin{array}{l}1 \\
\text { permanent }\end{array}$ & $2-40 \mathrm{mCi}$ & Preirradiated (24-56Gy) & & & & & \\
\hline $\begin{array}{l}\text { Ruge } 2011 \\
{[154]}\end{array}$ & $\begin{array}{l}90 \\
\text { temporary }\end{array}$ & $\begin{array}{l}\text { 50Gy } \\
\text { 3-6cGy/h }\end{array}$ & Metastases (90) & & & & & $\begin{array}{l}9 \\
\text { months }\end{array}$ \\
\hline \multirow[t]{3}{*}{$\begin{array}{l}\text { Suchorska } \\
2011[106]\end{array}$} & $\begin{array}{l}95 \\
\text { temporary }\end{array}$ & $50-65 G y$ & Astrocytomas II (69) & & & & & $\begin{array}{l}238 \\
\text { months }\end{array}$ \\
\hline & & $3 c G y / h$ & $\begin{array}{l}\text { Oligoastrocytomas II } \\
\text { (14) }\end{array}$ & & & & & \\
\hline & & $12 \mathrm{mCi}$ & $\begin{array}{l}\text { Oligodendrogliomas II } \\
\text { (12) }\end{array}$ & & & & & \\
\hline \multirow[t]{4}{*}{$\begin{array}{l}\text { Ruge } 2011 \\
\text { [199] }\end{array}$} & $\begin{array}{l}147 \\
\text { temporary }\end{array}$ & 65Gy & Children: & & & & & \\
\hline & & $1-31 \mathrm{mCi}$ & Astrocytomas I (101) & $100 \%$ & $98 \%$ & $93 \%$ & $82 \%$ & \\
\hline & & & Astrocytomas II (34) & & & $96 \%$ & $85 \%$ & \\
\hline & & & Other (12) & & & $85 \%$ & $77 \%$ & \\
\hline $\begin{array}{l}\text { Ruge } 2011 \\
\text { [163] }\end{array}$ & & 50Gy & Metastases & & & & & $\begin{array}{l}15 \\
\text { months }\end{array}$ \\
\hline
\end{tabular}


first-line treatment option for selected patients with highly eloquent tumour locations, bearing a high risk for open tumour resection. As pilocytic astrocytomas are mostly slow growing, sharply marginated tumours in the midline and highly eloquent areas, brachytherapy should be considered an attractive option.

\section{Brachytherapy of gliomas WHO grade II-IV}

Many more patients with gliomas WHO grade II-IV have been treated with iodine- 125 implants. However, the place of interstitial brachytherapy remains poorly defined. Moreover, the effects of an oligodendroglial differentiation, as well as recently discovered molecular genetic markers (such as LOH1p/19q, TP53 mutations, MGMT promoter methylation and IDH1 mutations), on the efficiency of interstitial brachytherapy, have not been evaluated yet [16].

\section{Brachytherapy of gliomas WHO grade II}

Results of brachytherapy for low grade gliomas WHO grade II - as first line treatment option - have been already summarized before [97]. Most data come from retrospective studies: 1-, 3- and 5-year survival rates in the range of $79-94 \%, 36-73 \%$ and $21-65 \%$ for primary tumours and 1and 3-year survival rates of around $68 \%$ and $30 \%$ for recurrent tumours have been reported $[4,12,25,67,68,93$, 95,98-101].

The response rates of astrocytomas WHO grade II for iodine-125 brachytherapy included 7.5-25.8\% complete responses, $13.8-29.0 \%$ partial responses, and $45.2-61.1 \%$ stable diseases. The rate of non-responders ranges between $0 \%$ and $17.6 \%[67,102]$.

One of the largest long-term analysis (median follow-up: 10.3 years) reports on interstitial brachytherapy in 239 patients with progressive eloquently located circumscribed, supratentorial WHO grade II gliomas [67]. In 103 patients permanent implants were used, since 1985 temporary implants have been preferred. Five-, 10-, and 15year progression free survival was $45 \%, 21 \%$, and $14 \%$, respectively. The corresponding survival rates were $51 \%$, $32 \%$, and $22 \%$, respectively. Of note, no levelling off of the Kaplan Meier curves was observed and patients experienced tumour progression even 10 years after treatment. Complete response was seen in 18 patients, partial response in 33 patients, tumour control in 146 patients, and unrestrained tumour progression in 42 patients (nonresponder group). Transient radiogenic complications occurred in 19 patients.

Schnell et al. found a 5-year survival rate as high as 93\% in patients with low activity temporary iodine-125 implants (54Gy, 10cGy/h) [102].

Malignant transformation of astrocytomas II was seen in $33 \%$ of the patients after 5 years, in 54\% after 10 years and in $67 \%$ after 15 years [67], but was not different to open tumour resection alone [16].

In a retrospective analysis, Warnke and co-workers observed a significant reduction of seizure incidence and increase of benzodiazepine receptor density (as demonstrated by single photon emission computed tomography in a subset of 20 patients), after brachytherapy of 80 patients with temporal WHO grade II astrocytomas [103]. Of note, $79 \%$ of patients became seizure free after six months.

For astrocytomas grade II, a significantly better outcome with less radiation toxicity was reached with temporary seeds than with permanent ones, which are associated with an increased risk for prolonged oedema [95,100,101,104] . Target volume and radiation dose showed a direct correlation with the risk of radionecrosis with critical values being $35 \mathrm{~cm}^{3}$ and 100Gy for permanent and 50Gy at $42 \mathrm{cGy} / \mathrm{h}$ for temporary implants [105]. Age, KPS, midline shift, tumour volume and enhancement on CT were predictors for outcome [68,95,99-101].

A combination of microsurgery and stereotactic brachytherapy for tumour remnants is feasible and might be an alternative treatment strategy, for example, for large lesions with significant proportions of eloquent tumour parts to avoid neurological deficits [16]. Brachytherapy to treat progressive or recurrent tumours after resection achieved a tumour response rate of $35.9 \%$ and a control rate of $97.3 \%$ after 24 months; median progression free survival was 53 months [106].

All in all, even though stereotactic brachytherapy has been used for low-grade gliomas for many years, its place within the multimodality treatment concept is still debated [16]. Small (less than $4 \mathrm{~cm}$ in diameter), circumscribed lesions in highly eloquent or deep-seated tumour locations might be good candidates for interstitial brachytherapy, especially in cases harbouring an increased risk for open tumour resection. Moreover, radiobiology, tumour shape and the option of percutaneous radiotherapy for recurrence, favour an increased application of brachytherapy in astrocytomas grade II.

\section{Brachytherapy of anaplastic gliomas WHO grade III}

For newly diagnosed anaplastic astrocytomas WHO grade III, standard therapy consists of radiation therapy with/-out surgical resection, whenever safely possible. Alternatively, first line chemotherapy might be considered (NOA-04 study). However, interstitial brachytherapy might also be a local treatment option, especially for those patients that are not clear surgical cases.

For newly diagnosed anaplastic astrocytomas, the combination of interstitial brachytherapy with percutaneous radiotherapy has been shown to be associated with 1-, 2-, 3 - and 5-year survival rates of 33-87\%, 36-75\%, 17-66\% and $0-50 \%$. Median survival is also highly variable and ranges between 8-57 months. Patients treated with seeds for recurrent anaplastic astrocytomas had 1-, 2- and 3year survival rates of $49-81 \%, 46-55 \%$ and $24-40 \%$ and a median survival of 9-35 months after implantation $[4,23,25,44,45,53,81,93,95,98,107-124]$. 
Two studies found an enhanced median survival in patients treated with percutaneous radiotherapy in combination with temporary iodine-125 implants, compared to external irradiation alone $[24,125]$. While in the NCOG 6G-82-2 study, the brachytherapy boost had no additional advantage [23].

Gutin et al. compared survival after iodine-125 brachytherapy for recurrent anaplastic astrocytomas to that of a historical chemotherapy group and obtained better survival data for the brachytherapy group [110].

Factors associated with improved survival were age $\leq$ 45-50 years, KPS $\geq 80-90$, superficial location, decreasing volume of implanted tumour, chemotherapy at recurrence and reoperation at the original site in case of tumour recurrence $[24,117,124,126]$.

Different schemes have been used for primary therapy. On the one hand, 50-60Gy of temporary implants have been combined with 50-60Gy percutaneous radiotherapy $[24,81,107]$. On the other hand, 100Gy of permanent implants have been added to 50-60Gy external beam radiotherapy [124]. Sometimes seeds had been implanted before percutaneous radiotherapy had been started [108].

There was as well a lot of variation in therapy for recurrent, pre-irradiated astrocytomas III. Doses of 50150 Gy have been employed with temporary implants [44,45,107,111,127]; 100-300Gy with permanent [53,128].

To summarize, multiple, even several randomized, studies were performed to analyze the value of brachytherapy in the primary treatment of astrocytomas grade III, but failed to prove any clear beneficial efficacy for this tumour entity. Therefore, standard therapy is radiotherapy or chemotherapy according to the results of the NOA-04 study [129]. However, for recurrent, pre-irradiated tumours, seeds might be a valuable option.

\section{Brachytherapy of glioblastomas WHO grade IV}

Interstitial brachytherapy has been repeatedly suggested for malignant gliomas WHO grade IV at primary diagnosis, as well as during the course of the disease. For primary glioblastomas, 1-, 2-, 3- and 5-year survival rates of 80$91 \%, 19-57 \%, 14-40 \%$ and $9-18 \%$ and a median survival of 13-26 months could be achieved. 1-, 2- and 3-year survival rates for recurrent tumours were $44-66 \%, 13-26 \%$ and 8$15 \%$, and median survival $6-17$ months $[4,23,25,44,45,53$, 81,95,108-119,121-123,128,130-142].

Several retrospective studies showed a better survival for patients with primary glioblastomas treated with a temporary or permanent brachytherapy boost, in addition to parallel or sequential external beam irradiation, instead of percutaneous radiotherapy alone (e.g. 1-, 2-, 3and 4-year survival rates have been $40 \%, 12.5 \%, 9 \%$ and $5 \%$ compared to $83-87 \%, 34-57 \%, 27 \%$ and $16 \%$; median survival 11 months vs. 18 months) [134,137]. There was no survival difference for iridium-192 and iodine-125
[139], or brachytherapy and radiosurgery boost [132], but permanent implants showed improved survival compared to temporary [115].

Percutaneous and interstitial irradiation in newly diagnosed paediatric malignant gliomas have been complemented by high-dose chemotherapy and autologous bone marrow rescue, but was not associated with better outcome scores than for conventional regimens [143].

For recurrent glioblastomas, tumour resection plus permanent seed implantation was compared to biopsy plus temporary seed implantation, and revealed similar median survival rates [133].

Subgroup analyses of the results for primary treatment of anaplastic astrocytomas and glioblastomas with percutaneous radiotherapy and permanent brachytherapy boost, divided into the RTOG recursive partitioning analysis (RPA) classes, have shown the following results: the 2-year survival rate and the median survival for RPA classes I/II were $68 \%$ and 37 months, for class III $74 \%$ and 28-31 months, for class IV $32-34 \%$ and 16 months and for classes V/VI $29 \%$ and 11 months. Iodine-125 has improved the survival most demonstrably in poorer prognostic classes so that a selection bias does not account for the survival benefit [121,122].

Age has an important effect on survival with a median survival of 109 weeks for patients aged 30-40 years, 96 weeks for 40-50 years, 77 weeks for 50-60 years and 76 weeks for 60 years and older [141]. Other factors affecting outcome were KPS, superficial lesion, gross total resection, reoperation, residual tumour volume, number of seeds, seed activity, volume implanted and dose $[25,117,126,140,142,144]$.

A recurrence pattern analysis of patients treated in the NCOG 6G-82-2 trial revealed that 77\% were local (within $2 \mathrm{~cm}$ from seed), 14\% separate, $5 \%$ subependymal and 5\% systemic [136]. Others found 35\% local, $65 \%$ marginal and $28 \%$ distant relapse [137] or $70 \%$ local, $18 \%$ distant and $12 \%$ both [128].

Many different dose combinations of percutaneous radiotherapy and iodine-125 brachytherapy have been given in the primary therapy setting; 45Gy whole brain radiotherapy and 100-280Gy (15-30cGy/h) brachytherapy boost [47], 59.4Gy (1.8Gy, 5×/week) or 40Gy (2Gy, $2 \times /$ day) and a high activity brachytherapy boost of $50 \mathrm{~Gy}$ [137], but most percutaneous and temporary seed dose were 60 Gy $[139,141]$. Sometimes radiotherapy was combined with chemotherapy regimens $[109,118,125,138]$ or hyperthermia [145], or was given after seed implantation [108].

For pre-irradiated, recurrent glioblastomas, seeds have been implanted only without further percutaneous radiotherapy. 100-500Gy of permanent seed dose have been chosen $[140,142]$ or $50-120 \mathrm{~Gy}$ of temporary $[44,110,111]$. 
All in all, multiple studies for newly diagnosed glioblastomas have been performed, but brachytherapy does not play a role in this setting nowadays. Standard therapy is surgery and radiotherapy, plus concomitant and adjuvant temozolomide [146]. In the recurrent setting, brachytherapy might be an option as interstitial irradiation can still be employed in cases in which tolerance of the healthy brain tissue has been reached because of previous external irradiation [4]. Interstitial irradiation offers excellent palliation in a majority of patients with a significant amount of long-term survivors for recurrent gliomas [147].

\section{Brachytherapy of brain metastases}

Besides primary brain tumours, interstitial brachytherapy should be considered for brain metastases [148,149]. Some accept a maximum diameter of $4 \mathrm{~cm}$ [150], others implant 3-6 cm metastases that are beyond the size limit of radiosurgery [151]. The advantages of treating brain metastases with brachytherapy are a minimally invasive procedure, a minimal damage to normal brain tissue, no supplementary risk by anaesthesia, no interference with other therapeutic modalities, low stress for the patient, a short hospitalisation time (2-3 days) and low costs. The reasons why brain metastases are ideal for interstitial irradiation are the frequent spherical shape, the mostly relatively small size, the normal brain parenchyma being displaced outside the potential target volume and the minimally invasive growth [152].

For metastases, local control rates of $73-95 \%$ have been reported [153,154].

Median survival after implantation varies from 4 to 18 months, and 12- and 18-months survival rates from 33 to $72 \%$ and from 25 to $33 \%[104,115,145,149-151,153$, 155-161].

Kreth et al. compared the median survival after iodine-125 brachytherapy (60Gy, 10cGy/h, temporary), percutaneous radiotherapy (40Gy, whole brain radiotherapy) and a combination of both, for newly diagnosed brain metastases, and observed median survival times of 12-15 months, 8 months and 17 months, respectively. For recurrent metastases, survival times were around 6 months $[104,150]$. Results for singular brain metastases are comparable with stereotactic radiosurgery [149].

For metastases, good prognostic factors are KPS $\geq 70$, solitary metastasis, no extracerebral metastases, long time interval between primary diagnosis and diagnosis of metastases [104,150,162].

A potential risk is growth of tumour cells by inoculation along the catheter pathway, which was seen by Ruge et al. in 6 out of 90 patients [154].

All in all, iodine-125 brachytherapy for metastases has become more popular again in recent years due to its radiobiological advantages and as it can be employed even after extensive percutaneous pre-irradiation [120], additionally biopsies are possible in the same session [154,163].

\section{Brachytherapy of pineal parenchymal tumours}

Iodine-125 seeds (50-60Gy, 9-12cGy/h) have been used for recurrent pineoblastomas [164]. A study of 18 patients (6-68 years) treated with 40-65Gy of permanent (11) or temporary (7) implants for primary pineal tumours or in a salvage situation has been published. 5 year overall actuarial survival rates of $100 \%$ for pineocytomas and of $78 \%$ for high-grade tumours have been reported. Complete remission rate was $72 \%$ and partial remission rate $28 \%$. Maarouf et al. concluded that iodine125 seeds are efficient and safe and an attractive alternative to microsurgery [165]. All in all, iodine-125 brachytherapy treatment with a low dose rate seems to be an attractive, minimally invasive treatment option of these rare tumour entities of the pineal region.

\section{Various indications of brachytherapy}

One group implanted petroclival meningiomas [26]. Treatment of meningiomas of the skull base and other localisations with permanent iodine-125 implants results in a complete response rate of $73-82 \%$ and a partial response rate of $18-27 \%$, without early, and with rare late complications [166-170]. The treatment of parasellar-clival and globoid meningiomas with permanent implants showed a slow reduction in volume with no mortality [171]. All these results are only of historical interest as seeds do not play a role in modern treatment of meningiomas.

Case reports present successful treatments of an example of bilateral blindness secondary to a recurrent, preirradiated hemangiopericytoma of the pituitary fossa [172], or of radiation-induced neoplasms [173]. Even a clival chordoma [174] and other skull base tumours [175,176] have been treated with an implantation of seeds, as well as locations that are a challenge for high-precision percutaneous radiotherapy [177,178]. A case of Rosai-Dorfman disease of the central nervous system has been cured with brachytherapy [179], as well as child optic pathway gliomas [180]. Craniopharyngiomas have been implanted without recurrences $[181,182]$ and recurrent medulloblastomas have been treated with iodine-125 brachytherapy [183]. Sometimes iodine-125 implants may have a favourable long-lasting effect upon medically refractory seizures [184].

\section{Side effects and complications}

The following side effects and complications have been described in the literature: intracranial pressure and tumour expansion due to necrosis, severe oedema, cerebral artery occlusion, intracranial haemorrhage and subdural bleeding, headaches, nausea, vomiting, seizures, increased neurological deficits, abscesses, aseptic and septic meningitis, wound infections and dehiscence, 
delayed wound healing, scalp infections, cerebrospinal fluid leakage, long-term steroid dependency, progressive dementia, psychotic symptoms, facial pain, pulmonary embolism and others $[12,13,22,24,45-47,70,81,101,107$, $110,114,134,137,138,155,185]$. Two cases of devastating strokes due to intracranial arterial occlusion have been reported [43]. Tacke et al. found in 6 out of 13 children with low-grade hypothalamic gliomas signs of vasculopathy in magnetic resonance imaging, but only one of them revealed symptoms of intermittent cerebral ischemia [186].

No toxicity was reported in $92 \%$, severe acute toxicity in $6 \%$, life threatening in $1 \%$ and fatal in $<1 \%$ [25]. All in all, the operative morbidity seems to be low (1.2-21.7\%) $[67,79,95,107,150,155]$. In patients with gliomas grade II, a combination of surgery plus iodine- 125 brachytherapy had a morbidity of $27.8 \%$, seed implantation alone of $6.4 \%$ [102]. The perioperative mortality rate is max. $0.8-2.6 \%$ $[4,67,68]$.

Radiation toxicity (oedema) has been reported mostly among patients treated with permanent implants $[101,104]$. It occurs in $1.7-7.5 \%[68,95,96,101,104]$, although for tumours greater than $3.5 \mathrm{~cm}$ in diameter, it occurs in up to $18 \%$ of cases [101]. Late radiation necrosis is a severe side effect which seems to occur in $40 \%$ of the low-dose-rate implantations and is correlated with total radiation dose, implanted activity and the velocity of tumour shrinkage. It can be avoided by the usage of temporary implants [85]. A detailed analysis of patients with grade I and grade II gliomas undergoing low-activity iodine-125 brachytherapy has revealed as risk factors for radiogenic complications the volume of intratumoural $200 \mathrm{~Gy}$ isodose and rapid tumour shrinkage of $\geq 50 \%$ of the volume in six months. A 200Gy-isodose-volume of < $4.5 \mathrm{ml}$ leads to $<3 \%$ radiogenic complications with a steep increase of the risk beyond this limit. For temporary implants with a reference dose of 60Gy, the risk for complications is $<3 \%$ if the treatment volume is $\leq 23 \mathrm{ml}$. Further factors affecting the toxicity rate examined are the overall treatment volume, the volume of the 60Gy isodose outside the target volume, reimplantation, the reference dose, the number of implanted seeds and a lobar tumour location [96]. Other authors identified a target volume > $35 \mathrm{ml}$ and a permanent seed dose $>100 \mathrm{~Gy}$, or a temporary $>50 \mathrm{~Gy}$, as risk factors of complications [105]. Evaluation of a brain necrosis after permanent seed implantation showed the necrotic area within the 100Gy isodose and damage to the blood-brain barrier within the 50Gy isodose [187]. A long term analysis of Kreth et al. revealed a risk for radiogenic complications of about $9 \%$ for relatively small grade II gliomas (diameter $<4 \mathrm{~cm}$ ) and a steep increase of complications (about 25\%) for those harbouring larger tumours $(\geq 4 \mathrm{~cm})$. The increase of radiogenic complication rate beyond a critical threshold can be explained by the tissue effects of brachytherapy, such as the increase of capillary permeability in the vicinity of the high-dose zone and the exponential increase of the damaged capillary surface area product with the square of the radius of the high-dose zone [16]. The risk of low-dose rate brachytherapy of low-grade gliomas should not be confused with the high frequency of complications (40$50 \%$ ) after high-dose rate treatment of malignant gliomas. It remains difficult to elucidate, in which extent, additional external beam radiation, the often relatively large size of the treatment volume, and the applied high dose rate (in the range of 35-50cGy/h), have contributed to these high complication rates. Reoperation has been shown to be required in $0-72 \%$ because of treatment induced space occupying lesions, which occurred particularly after high dose rate brachytherapy 3-183 weeks after implantation $[23,25,44-46,53,79,81,95,101,104,105,107,109-114,116-119$ ,123,124,127,130,132,133,135-139,144,150,155,185,188,189]. No correlation between implanted volume and need for reoperation has been found [137]. However, there might be a correlation for the combination of percutaneous radiotherapy plus brachytherapy (64\%), compared to percutaneous radiotherapy only (15\%) [137], others found similar rates of around 30\% for both groups [24]. Histological findings (necrosis, tumour) at reoperation are not prognostic factors [190].

Especially permanent seeds and periventricular location, carry the risk of seed migration $[133,191]$ but temporary seeds also sometimes need repositioning $[46,120]$.

Percutaneous catheter derived brachytherapy may be associated with an increased incidence of extraneural metastatic gliomas of the scalp, skull, cervical nodes, etc. [192].

\section{Quality of life}

The quality of life in the majority of long-term survivors appears to be quite satisfactory $[23,44,45,193]$. Comparing permanent and temporary implants, the KPS remained stable or improved in $85 \%$ and $58 \%$. The neurological status emended in $87 \%$ and $67 \%$ [115]. In another study the neurological function has improved in $30 \%$, has shown no change in $43 \%$ and has declined in $27 \%$ [95]. The KPS mostly improved in patients with astrocytomas II [104].

\section{Summary and proposals for reduced side effects}

In summary, to reduce the risk for side effects, temporary implants, dose rates around 10cGy/h and activities < $20 \mathrm{mCi}$ should be preferred, high dose zones > 150Gy should not be located within normal tissue and next to vessels, and volumes $>4 \mathrm{~cm}$ should not be implanted. If these guidelines are followed, then side effects are very rare [16].

\section{Conclusions}

Iodine-125 brachytherapy might be indicated in highly selected patients with recurrent brain tumours as a form 
of re-irradiation, low-grade gliomas, brain metastases with stable systemic disease and some other less common diagnoses, as it is a way to deliver an additional dose of localized radiation to malignant brain tumours while limiting radiation to the surrounding normal brain tissue [13]. However, there is no strong randomized evidence that would justify the change of guidelines for any brain tumour entity. The side effects are rather rare. During follow-up, attention has to be paid to some special diagnostic features. In that case, iodine-125 seed implantation in brain tumours may lead to good survival rates and a decent quality of life.

\section{Author details}

'Department of Radiation Oncology, Ludwig-Maximilians-University Hospital, Marchioninistr. 15, 81377 Munich, Germany. '2Department of Neurosurgery, Ludwig-Maximilians-University Hospital, Marchioninistr. 15, 81377 Munich, Germany.

\section{Authors' contributions}

SBS designed the protocol, conducted data evaluation and wrote the article. NT also wrote the article. KN, MN, JCT, CB and FWK performed a critical review of the manuscript. CB and FWK also designed the protocol. All authors read and approved the final manuscript.

\section{Competing interests}

The authors declare that they have no competing interests.

Received: 5 January 2012 Accepted: 6 March 2012

Published: 6 March 2012

\section{References}

1. Holm HH, Stroyer I, Hansen H, Stadil F: ULTRASONICALLY GUIDED PERCUTANEOUS INTERSTITIAL IMPLANTATION OF I125 SEEDS IN CANCER-THERAPY. British Journal of Radiology 1981, 54(644):665-670.

2. Matthews CME: HALF-LIFE OF IODINE-125. Physics in Medicine and Biology $1960,5(1): 45-47$

3. Kim JH, Hilaris B: IODINE 125 SOURCE IN INTERSTITIAL TUMOR THERAPY CLINICAL AND BIOLOGIC CONSIDERATIONS. American Journal of Roentgenology 1975, 123(1):163-169.

4. Mundinger F, Ostertag CB, Birg W, Weigel K: STEREOTACTIC TREATMENT OF BRAIN-LESIONS - BIOPSY, INTERSTITIAL RADIOTHERAPY (IR-192 AND I125) AND DRAINAGE PROCEDURES. Applied Neurophysiology 1980, 43(35):198-204.

5. Bernstein M, Gutin PH: Interstitial irradiation of brain tumors: a review. Neurosurgery 1981, 9(6):741-750.

6. Jani SK, Hitchon PW, Vangilder JC, Wen BC: NORMAL BRAIN IRRADIATION DURING STEREOTACTIC BRAIN IMPLANTS USING RADIOACTIVE I-125. Applied Neurophysiology 1987, 50(1-6):310-313.

7. Viola A, Major T, Julow J: Comparison of $\mathbf{I}-125$ stereotactic brachytherapy and LINAC radiosurgery modalities based on physical dose distribution and radiobiological efficacy. Radiation Research 2006, 165(6):695-702.

8. Luxton G, Petrovich Z, Jozsef G, Nedzi LA, Apuzzo ML: Stereotactic radiosurgery: principles and comparison of treatment methods. Neurosurgery 1993, 32(2):241-259, discussion 259.

9. Larson DA, Flickinger JC, Loeffler JS: The radiobiology of radiosurgery. Int J Radiat Oncol Biol Phys 1993, 25(3):557-561.

10. Larsson B: Radiobiological Fundamentals in Radiosurgery. Radiosurgery 1992, 3-14.

11. Krishnaswamy V: DOSE DISTRIBUTION AROUND AN 1125 SEED SOURCE IN TISSUE. Radiology 1978, 126(2):489-491.

12. Sneed PK, McDermott MW, Gutin PH: Interstitial brachytherapy procedures for brain tumors. Seminars in Surgical Oncology 1997, 13(3):157-166.

13. McDermott MW, Sneed PK, Gutin PH: Interstitial brachytherapy for malignant brain tumors. Seminars in Surgical Oncology 1998, 14(1):79-87.
14. Nath R, Anderson LL, Luxton G, Weaver KA, Williamson JF, Meigooni AS: DOSIMETRY OF INTERSTITIAL BRACHYTHERAPY SOURCES RECOMMENDATIONS OF THE AAPM RADIATION-THERAPY COMMITTEE TASK GROUP NO 43. Medical Physics 1995, 22(2):209-234

15. Sewchand W, Drzymala RE, Amin PP, Salcman M, Salazar OM: RADIATION CONTROL IN THE INTENSIVE-CARE UNIT FOR HIGH-INTENSITY IR-192 BRAIN IMPLANTS. Neurosurgery 1987, 20(4):584-588.

16. Kreth FW, Thon N, Siefert A, Tonn JC: The place of interstitial brachytherapy and radiosurgery for low-grade gliomas. Adv Tech Stand Neurosurg 2010, 35:183-212.

17. Mundinger F: [Stereotaxic interstitial therapy of non-resectable intracranial tumors with iridium-192 and iodine-125]. Strahlentherapie [Sonderb] 1981, 76:90-112.

18. Fike JR, Cann CE, Phillips TL, Bernstein M, Gutin PH, Turowski K, Weaver KA, Davis RL, Higgins RJ, Dasilva V: Radiation Brain-Damage Induced by Interstitial 1125 Sources - a Canine Model Evaluated by Quantitative Computed-Tomography. Neurosurgery 1985, 16(4):530-537.

19. Groothuis DR, Wright DC, Ostertag CB: The Effect of I-125 Interstitial Radiotherapy on Blood-Brain-Barrier Function in Normal Canine Brain. $J$ Neurosurg 1987, 67(6):895-902.

20. Ostertag CB: Brachytherapy - Interstitial Implant Radiosurgery. Acta Neurochir 1993, 79-84.

21. Meli JA, Dicker CS, Schulz RJ: COMPUTER-APPLICATIONS TO RADIOACTIVESEED - BRAIN-TUMOR IMPLANTS. Computerized Medical Imaging and Graphics 1989, 13(3):269-274.

22. Hosobuchi Y, Phillips TL, Stupar TA, Gutin PH: INTERSTITIAL BRACHYTHERAPY OF PRIMARY BRAIN-TUMORS - PRELIMINARY-REPORT. J Neurosurg 1980, 53(5):613-617.

23. Gutin PH, Prados MD, Phillips TL, Wara WM, Larson DA, Leibel SA, Sneed PK, Levin VA, Weaver KA, Silver $P$, et al: EXTERNAL IRRADIATION FOLLOWED BY AN INTERSTITIAL HIGH-ACTIVITY I-125 IMPLANT BOOST IN THE INITIAL TREATMENT OF MALIGNANT GLIOMAS - NCOG STUDY 6G-82-2. International Journal of Radiation Oncology Biology Physics 1991, 21(3):601-606

24. Laperriere NJ, Leung PMK, McKenzie S, Milosevic M, Wong S, Glen J, Pintilie M, Bernstein M: Randomized study of brachytherapy in the initial management of patients with malignant astrocytoma. International Journal of Radiation Oncology Biology Physics 1998, 41(5):1005-1011.

25. Scharfen CO, Sneed PK, Wara WM, Larson DA, Phillips TL, Prados MD, Weaver KA, Malec M, Acord P, Lamborn KR, et al: HIGH-ACTIVITY I-125 INTERSTITIAL IMPLANT FOR GLIOMAS. International Journal of Radiation Oncology Biology Physics 1992, 24(4):583-591.

26. Kumar PP, Good RR, Patil AA, Leibrock LG: PERMANENT HIGH-ACTIVITY I125 IN THE MANAGEMENT OF PETROCLIVAL MENINGIOMAS - CASEREPORTS. Neurosurgery 1989, 25(3):436-442.

27. Ling CC: GOLD-198 PALLADIUM-103 AND IODINE-125 PERMANENT IMPLANTS RADIOBIOPHYSICAL CONSIDERATIONS. Medical Physics (Woodbury) 1991, 18(3):637

28. Bauerkirpes B, Sturm V, Schlegel W, Lorenz WJ: COMPUTERIZED OPTIMIZATION OF I125 IMPLANTS IN BRAIN-TUMORS. International Journal of Radiation Oncology Biology Physics 1988, 14(5):1013-1023.

29. Meningher $H$, Benary $V$, Chaitchick S, Akselrod S: A PLANNING METHOD FOR I-125 IMPLANTS IN CANCER-THERAPY. Physics in Medicine and Biology 1990, 35(12):1633-1640

30. Rosenow UF, Findlay PA, Wright DC: THE NCI-ATLAS OF DOSE DISTRIBUTIONS FOR REGULAR I-125 BRAIN IMPLANTS. Radiotherapy and Oncology 1987, 10(2):127-139.

31. Saw CB, Suntharalingam N, Ayyangar KM, Tupchong L: DOSIMETRIC CONSIDERATIONS OF STEREOTACTIC BRAIN IMPLANTS. International Journal of Radiation Oncology Biology Physics 1989, 17(4):887-891.

32. Weaver K, Smith $V$, Lewis JD, Lulu B, Barnett CM, Leibel SA, Gutin P, Larson D, Phillips T: A CT-BASED COMPUTERIZED TREATMENT PLANNING SYSTEM FOR I-125 STEREOTACTIC BRAIN IMPLANTS. International Journal of Radiation Oncology Biology Physics 1990, 18(2):445-454.

33. Zamorano L, Dujovny M, Malik G, Yakar D, Mehta B: MULTIPLANAR CTGUIDED STEREOTAXIS AND I125 INTERSTITIAL RADIOTHERAPY - IMAGEGUIDED TUMOR VOLUME ASSESSMENT, PLANNING, DOSIMETRIC CALCULATIONS, STEREOTACTIC BIOPSY AND IMPLANTATION OF REMOVABLE CATHETERS. Applied Neurophysiology 1987, 50(1-6):281-286.

34. Ahmad M, Fontenla DP, Tsao STC, Chui CS, Reiff JE, Anderson LL, Huang DYC, Schell MC: DIODE DOSIMETRY OF MODEL-6711 AND MODEL- 
6712 I-125 SEEDS IN A WATER PHANTOM. Medical Physics 1992, 19(2):391-399.

35. Dale RG: SOME THEORETICAL DERIVATIONS RELATING TO THE TISSUE DOSIMETRY OF BRACHYTHERAPY NUCLIDES, WITH PARTICULAR REFERENCE TO I125. Medical Physics 1983, 10(2):176-183.

36. Hartmann GH, Schlegel W, Scharfenberg H: THE 3-DIMENSIONAL DOSE DISTRIBUTION OF I-125 SEEDS IN TISSUE. Physics in Medicine and Biology 1983, 28(6):693-699.

37. Krishnaswamy V: DOSE TABLES FOR 1125 SEED IMPLANTS. Radiology 1979, 132(3):727-730.

38. Mainegra E, Capote R, Lopez E: Dose rate constants for I-125, Pd-103, Ir192 and Yb-169 brachytherapy sources: an EGS4 Monte Carlo study. Physics in Medicine and Biology 1998, 43(6):1557-1566.

39. Mobit P, Badragan I: An evaluation of the AAPM-TG43 dosimetry protocol for I-125 brachytherapy seed. Physics in Medicine and Biology 2004, 49(14), DOI 10.1088/0031-9155/1049/1014/1010|PII S0031-9155(1004) 72823-72827.

40. Rodriguez EAV, Alcon EPQ, Rodriguez ML, Gutt F, de Almeida E: Dosimetric parameters estimation using PENELOPE Monte-Carlo simulation code: Model 6711 a I-125 brachytherapy seed. Applied Radiation and Isotopes 2005, 63(1):41-48.

41. Anderson LL, Harrington PJ, Osian AD, Arbit E, Leibel SA, Malkin MG: A VERSATILE METHOD FOR PLANNING STEREOTAXIC BRAIN IMPLANTS. Medical Physics 1993, 20(5):1457-1464.

42. Kall BA, Kelly PJ, Stiving SO, Goerss SJ: Preoperative computer-assisted simulation of interstitial iodine-125 source placements into CNS lesion volumes. Stereotactic and Functional Neurosurgery 1992, 59(1-4):205.

43. Bernstein M, Lumley M, Davidson G, Laperriere N, Leung P: INTRACRANIAL ARTERIAL-OCCLUSION ASSOCIATED WITH HIGH-ACTIVITY I-125 BRACHYTHERAPY FOR GLIOBLASTOMA. J Neuro-Oncol 1993, 17(3):253-260.

44. Leibel S, Gutin P, Wara W, Silver P, Hannigan J, Lamb S, Weaver K, Phillips T: SURVIVAL AND QUALITY OF LIFE FOLLOWING INTERSTITIAL IMPLANTATION OF REMOVABLE HIGH-ACTIVITY IODINE-125 SOURCES FOR RECURRENT MALIGNANT GLIOMAS. International Journal of Radiation Oncology, Biology, Physics 1988, 15(SUPPL. 1):155-156.

45. Leibel SA, Gutin PH, Wara WM, Silver PS, Larson DA, Edwards MSB, Lamb SA, Ham B, Weaver KA, Barnett C, et al: SURVIVAL AND QUALITY OF LIFE AFTER INTERSTITIAL IMPLANTATION OF REMOVABLE HIGH-ACTIVITY I-125 SOURCES FOR THE TREATMENT OF PATIENTS WITH RECURRENT MALIGNANT GLIOMAS. International Journal of Radiation Oncology Biology Physics 1989, 17(6):1129-1139.

46. Willis BK, Heilbrun MP, Sapozink MD, McDonald PR: STEREOTACTIC INTERSTITIAL BRACHYTHERAPY OF MALIGNANT ASTROCYTOMAS WITH REMARKS ON POSTIMPLANTATION COMPUTED TOMOGRAPHIC APPEARANCE. Neurosurgery 1988, 23(3):348-354

47. Wright DC, Findlay P, Rosenow U, Orr K, Miller R, Harrington F, Kinsella T: IODINE-125 INTERSTITIAL BRACHYTHERAPY FOR PRIMARY MALIGNANT BRAIN TUMORS A PHASE I-II STUDY. International Journal of Radiation Oncology, Biology, Physics 1987, 13(SUPPL. 1):148-149.

48. Schupak KD, Fass D, Malkin M, Arbit E, Anderson L, Lindsley K, Leibel S: RELATIONSHIP OF THE PATTERNS OF RECURRENCE TO THE TECHNICAL ACCURACY OF STEREOTACTIC INTERSTITIAL IMPLANTATION FOR HIGH GRADE GLIOMAS. International Journal of Radiation Oncology, Biology, Physics 1991, 21(SUPPL. 1):221-222.

49. Eddy M, Deutsch M, Selker R: QUALITY ASSURANCE FOR IODINE-125 BRAIN IMPLANTS PROGRAM DESCRIPTION AND PRELIMINARY RESULTS. Medical Physics (Woodbury) 1991, 18(3):605.

50. Dale RG: THE APPLICATION OF THE LINEAR-QUADRATIC DOSE-EFFECT EQUATION TO FRACTIONATED AND PROTRACTED RADIOTHERAPY. British Journal of Radiology 1985, 58(690):515-528.

51. Dale RG: RADIOBIOLOGICAL ASSESSMENT OF PERMANENT IMPLANTS USING TUMOR REPOPULATION FACTORS IN THE LINEAR-QUADRATIC MODEL. British Journal of Radiology 1989, 62(735):241-244.

52. Dale RG, Jones B, Coles IP: EFFECT OF TUMOR SHRINKAGE ON THE BIOLOGICAL EFFECTIVENESS OF PERMANENT BRACHYTHERAPY IMPLANTS. British Journal of Radiology 1994, 67(799):639-645.

53. Gaspar LE, Zamorano L, Shamsa F, Fontanesi J, Ezzell GE, Yakar DA: Permanent (125)iodine implants for recurrent malignant gliomas. International Journal of Radiation Oncology Biology Physics 1999, 43(5):977-982.
54. Yaes RJ: Late normal tissue injury from permanent interstitial implants. International Journal of Radiation Oncology Biology Physics 2001, 49(4):1163-1169.

55. Julow J, Szeifert GT, Balint K, Nyary I, Nemes Z: The role of microglia/ macrophage system in the tissue response to $1-125$ interstitial brachytherapy of cerebral gliomas. Neurological Research 2007, 29(3):233-238.

56. Janzer RC, Kleihues P, Ostertag CB: Early and Late Effects on the Normal Dog Brain of Permanent Interstitial Ir-192 Irradiation. Acta Neuropathol 1986, 70(2):91-102.

57. Lehnert $\mathrm{S}$, Reniers $\mathrm{B}$, Verhaegen $\mathrm{F}$ : Relative biologic effectiveness in terms of tumor response of $1-125$ implants compared with Co-60 gamma rays. International Journal of Radiation Oncology Biology Physics 2005, 63(1):224-229.

58. Ling CC, Li WX, Anderson LL: THE RELATIVE BIOLOGICAL EFFECTIVENESS OF I-125 AND PD-103. International Journal of Radiation Oncology Biology Physics 1995, 32(2):373-378.

59. Zeitz L, Kim SH, Kim JH: RELATIVE BIOLOGICAL EFFECTIVENESS (RBE) OF X-RAYS SIMULATING RADIATION FROM ENCAPSULATED I125 SOURCES. Radiation Research 1976, 67(3):549-549.

60. Da Silva VF, Gutin PH, Deen DF, Weaver KA: RELATIVE BIOLOGICAL EFFECTIVENESS OF IODINE-125 SOURCES IN A MURINE BRACHYTHERAPY MODEL. International Journal of Radiation Oncology, Biology, Physics 1984, 10(11):2109-2112.

61. Siddiqi SN, Provias J, Laperriere N, Bernstein M: Effects of iodine-125 brachytherapy on the proliferative capacity and histopathological features of glioblastoma recurring after initial therapy. Neurosurgery 1997, 40(5):910-917.

62. Cron GO, Beghein N, Crokart N, Chavee E, Bernard S, Vynckier S, Scalliet P, Gallez B: Changes in the tumor microenvironment during low-dose-rate permanent seed implantation iodine-125 brachytherapy. International Journal of Radiation Oncology Biology Physics 2005, 63(4):1245-1251.

63. Julow J, Szeifert GT, Balint K, Nyary I, Nemes Z: Tissue response to iodine125 interstitial brachytherapy of cerebral gliomas. Prog Neurol Surg 2007, 20:312-323.

64. Fike JR, Cann CE, Bernstein M, Davis RL, Phillips TL, Gutin PH, Weaver KA: RADIATION-DAMAGE TO THE CANINE BRAIN AFTER I-125 IMPLANTATION. Radiation Research 1982, 91(2):370-370.

65. Kunz M, Thon N, Eigenbrod S, Hartmann C, Geisler J, Kretzschmar H, von Deimling A, Popperl G, Tonn J, Kreth F: Hot Spots in 18 Fet-Pet Delineate Malignant Tumor Parts within Suspected Who Grade li Glioma. NeuroOncology 2010, 12:12-12.

66. Kunz M, Thon N, Eigenbrod S, Hartmann C, Egensperger R, Herms J Geisler J, la Fougere C, Lutz J, Linn J, et al: Hot spots in dynamic (18)FETPET delineate malignant tumor parts within suspected WHO grade II gliomas. Neuro Oncol 2011, 13(3):307-316.

67. Kreth FW, Faist M, Grau S, Ostertag CB: Interstitial I-125 radiosurgery of supratentorial De Novo WHO grade 2 astrocytorna and oligoastrocytorna in adults - Long-term results and prognostic factors. Cancer 2006, 106(6):1372-1381.

68. Kreth FW, Faist M, Warnke PC, Rossner R, Volk B, Ostertag CB: INTERSTITIAL RADIOSURGERY OF LOW-GRADE GLIOMAS. I Neurosurg 1995, 82(3):418-429.

69. Findlay PA, Wright DC, Harrington FS, Miller RW, Glatstein E: IODINE-125 INTERSTITIAL BRACHYTHERAPY FOR PRIMARY MALIGNANT BRAIN TUMORS TECHNICAL ASPECTS OF TREATMENT PLANNING AND IMPLANTATION METHODS. International Journal of Radiation Oncology, Biology, Physics 1984, 10(SUPPL. 2):93-94.

70. Gutin PH, Phillips TL, Wara WM, Leibel SA, Hosobuchi Y, Levin VA, Weaver KA, Lamb S: BRACHYTHERAPY OF RECURRENT MALIGNANT BRAIN-TUMORS WITH REMOVABLE HIGH-ACTIVITY I125 SOURCES. J Neurosurg 1984, 60(1):61-68.

71. Julow J, Major T, Emri M, Valalik I, Sagi S, Mangel L, Nemeth G, Tron L, Varallyay G, Solymosi D, et al: The application of image fusion in stereotactic brachytherapy of brain tumours. Acta Neurochir 2000, 142(11):1253-1258

72. Peraud A, Goetz C, Siefert A, Tonn JC, Kreth FW: Interstitial iodine-125 radiosurgery alone or in combination with microsurgery for pediatric patients with eloquently located low-grade glioma: a pilot study. Childs Nervous System 2007, 23(1):39-46. 
73. Ten Haken RK, Diaz RF, McShan DL, Fraass BA, Taren JA, Hood TW: FROM MANUAL TO 3-D COMPUTERIZED TREATMENT PLANNING FOR IODINE125 STEREOTACTIC BRAIN IMPLANTS. International Journal of Radiation Oncology, Biology, Physics 1988, 15(2):467-480.

74. Viola A, Major T, Julow J: The importance of postoperative CT image fusion verification of stereotactic interstitial irradiation for brain tumors. International Journal of Radiation Oncology Biology Physics 2004, 60(1):322-328.

75. Knuefermann $\mathrm{H}$, Wannenmacher $\mathrm{M}$, Sommerkamp $\mathrm{H}$, Fiedler $\mathrm{L}$, Brobmann G: INDICATIONS AND LIMITS OF INTERSTITIAL RADIO THERAPY WITH IODINE-125 SEEDS. J Cancer Res Clin 1984, 107(SUPPL):S60.

76. Loeffler JS, Kooy HM, Wen PY, Fine HA, Cheng CW, Mannarino EG, Tsai JS, Alexander E: THE TREATMENT OF RECURRENT BRAIN METASTASES WITH STEREOTACTIC RADIOSURGERY. Journal of Clinical Oncology 1990, 8(4):576-582.

77. Healey EA, Shamberger RC, Grier HE, Loeffler JS, Tarbell NJ: A 10-YEAR EXPERIENCE OF PEDIATRIC BRACHYTHERAPY. International Journal of Radiation Oncology Biology Physics 1995, 32(2):451-455.

78. Stowe SM, Littman P, Wara W, Raney RB, Tefft M: THE USE OF IMPLANTATION IN CHILDHOOD TUMORS - THE EXPERIENCE OF CHILDRENS CANCER STUDY-GROUP MEMBER INSTITUTIONS. American Journal of Clinical Oncology-Cancer Clinical Trials 1982, 5(2):129-129.

79. Bernstein M, Laperriere N, Glen J, Leung P, Thomason C, Landon AE: BRACHYTHERAPY FOR RECURRENT MALIGNANT ASTROCYTOMA. International Journal of Radiation Oncology Biology Physics 1994, 30(5):1213-1217.

80. Frank F, Fabrizi AP, Gaist G, Frankricci R, Piazzi M, Spagnolli F: LATE CONSIDERATIONS IN THE TREATMENT OF LOW-GRADE MALIGNANCY CEREBRAL-TUMORS WITH I-125 BRACHYTHERAPY. Applied Neurophysiology 1987, 50(1-6):302-309.

81. Larson DA, Gutin PH, Leibel SA, Phillips TL, Sneed PK, Wara WM: STEREOTAXIC IRRADIATION OF BRAIN-TUMORS. Cancer 1990, 65(3):792-799.

82. Voges J, Gaus C, Schlegel W, Pastyr O, Wowra B, Sturm V: INTERSTITIAL IRRADIATION OF A LARGE, LOW-GRADE EPENDYMOMA WITH STEREOTAXICALLY IMPLANTED I-125 SEEDS. Acta Neurochir 1993, 122(12):127-129.

83. Mundinger F, Braus DF, Krauss JK, Birg W: LONG-TERM OUTCOME OF 89 LOW-GRADE BRAIN-STEM GLIOMAS AFTER INTERSTITIAL RADIATIONTHERAPY. J Neurosurg 1991, 75(5):740-746.

84. Julow J, Viola A, Major T, Valalik I, Sagi S, Mangel L, Kovacs BR, Repa I, Bajzik G, Zoltan TN, et al: lodine-125 brachytherapy of brain stem tumors. Strahlenther Onkol 2004, 180(7):449-454.

85. Wowra B, Schmitt HP, Sturm V: INCIDENCE OF LATE RADIATION NECROSIS WITH TRANSIENT MASS EFFECT AFTER INTERSTITIAL LOW-DOSE RATE RADIOTHERAPY FOR CEREBRAL GLIOMAS. Acta Neurochir 1989, 99(34):104-108.

86. Julow J, Kolumban Z, Viola A, Major T, Kolumban G: Prediction of volumetric change in the "triple ring" caused by glioma I-125 brachytherapy. Neuro-Oncology 2008, 10(4):583-592.

87. Voges J, Herholz K, Holzer T, Wurker M, Bauer B, Pietrzyk U, Treuer H, Schroder R, Sturm V, Heiss WD: C-11-methionine and F-18-2-fluorodeoxyglucose positron emission tomography: A tool for diagnosis of cerebral glioma and monitoring after brachytherapy with $1-125$ seeds. Stereotactic and Functional Neurosurgery 1997, 69(1-4):129-135.

88. Wurker M, Herholz K, Voges J, Pietrzyk U, Treuer H, Bauer B, Sturm V, Heiss WD: Glucose consumption and methionine uptake in low-grade gliomas after iodine-125 brachytherapy. European Journal of Nuclear Medicine 1996, 23(5):583-586.

89. Aiken AH, Chang SM, Larson D, Butowski N, Cha S: LONGITUDINAL MAGNETIC RESONANCE IMAGING FEATURES OF GLIOBLASTOMA MULTIFORME TREATED WITH RADIOTHERAPY WITH OR WITHOUT BRACHYTHERAPY. International Journal of Radiation Oncology Biology Physics 2008, 72(5):1340-1346.

90. Aarsen FK, Paquier PF, Reddingius RE, Streng IC, Arts WF, Evera-Preesman M, Catsman-Berrevoets CE: Functional outcome after low-grade astrocytoma treatment in childhood. Cancer 2006, 106(2):396-402.

91. Steinbok P: Management and outcome of low-grade astrocytomas of the midline in children: A retrospective review. Neurosurgery 1994, 35(2):342-343.

92. Sutton LN, Molloy PT, Sernyak H, Goldwein J, Phillips PL, Rorke LB, Moshang T Jr, Lange B, Packer RJ: Long-term outcome of hypothalamic/ chiasmatic astrocytomas in children treated with conservative surgery. $J$ Neurosurg 1995, 83(4):583-589.

93. Etou A, Mundinger F, Mohadjer M, Birg W: STEREOTACTIC INTERSTITIAL IRRADIATION OF DIENCEPHALIC TUMORS WITH IR-192 AND I-125 - 10 YEARS FOLLOW-UP AND COMPARISON WITH OTHER TREATMENTS. ChildS Nervous System 1989, 5(3):140-143.

94. Herrera EJ, Viano JC, Gomez JM, Surur A, Suarez JC: Interstitial stereotactic radiosurgery of pilocytic astrocytomas in paediatric patients. Acto Neurochir 2007, 149(9):887-896.

95. Ostertag CB, Kreth FW: I-125 INTERSTITIAL IRRADIATION FOR CEREBRAL GLIOMAS. Acta Neurochir 1992, 119(1-4):53-61.

96. Kreth FW, Faist M, Rossner R, Birg W, Volk B, Ostertag CB: The risk of interstitial radiotherapy of low-grade gliomas. Radiotherapy and Oncology 1997, 43(3):253-260

97. Vitaz TW, Warnke PC, Tabar V, Gutin PH: Brachytherapy for brain tumors. J Neurooncol 2005, 73(1):71-86

98. Julow J, Viola A, Balint K, Szeifert GT: Image fusion-guided stereotactic iodine-125 interstitial irradiation of inoperable and recurrent gliomas. Prog Neurol Surg 2007, 20:303-311.

99. Kreth FW, Faist M, Rossner R, Volk B, Ostertag CB: Supratentorial world health organization grade 2 astrocytomas and oligoastrocytomas - A new pattern of prognostic factors. Cancer 1997, 79(2):370-379.

100. Kreth FW, Warnke PC, Ostertag CB: INTERSTITIAL RADIOSURGERY IN TREATMENT OF LOW-GRADE GLIOMAS. Nervenarzt 1993, 64(10):633-639.

101. Mehrkens JH, Kreth FW, Muacevic A, Ostertag CB: Long term course of WHO grade II astrocytomas of the Insula of Reil after I-125 interstitial irradiation. J Neurol 2004, 251(12):1455-1464.

102. Schnell O, Scholler K, Ruge M, Siefert A, Tonn JC, Kreth FW: Surgical resection plus stereotactic $1-125$ brachytherapy in adult patients with eloquently located supratentorial WHO grade II glioma - Feasibility and outcome of a combined local treatment concept. J Neurol 2008, 255(10):1495-1502.

103. Warnke PC, Berlis A, Weyerbrock A, Ostertag CB: Significant reduction of seizure incidence and increase of benzodiazepine receptor density after interstitial radiosurgery in low-grade gliomas. Acta Neurochir Supp/ 1997, 68:90-92.

104. Kreth FW, Warnke PC, Ostertag CB: INTERSTITIAL IMPLANT RADIOSURGERY FOR CEREBRAL METASTASES. Acta Neurochir 1993, 112-114.

105. Scerrati M, Montemaggi P, lacoangeli M, Roselli R, Rossi GF: INTERSTITIAL BRACHYTHERAPY FOR LOW-GRADE CEREBRAL GLIOMAS - ANALYSIS OF RESULTS IN A SERIES OF 36 CASES. Acta Neurochir 1994, 131(1-2):97-105.

106. Suchorska B, Ruge M, Treuer H, Sturm V, Voges J: Stereotactic brachytherapy of low-grade cerebral glioma after tumor resection. Neuro Oncol 2011, 13(10):1133-1142.

107. Bernstein M, Laperriere N, Leung P, McKenzie S: INTERSTITIAL BRACHYTHERAPY FOR MALIGNANT BRAIN-TUMORS - PRELIMINARYRESULTS. Neurosurgery 1990, 26(3):371-380.

108. Clark WC, Fontanesi J, Patterson K, Kun L, Robertson JT: CT-GUIDED STEREOTACTIC BRACHYTHERAPY WITH IODINE-125 SEEDS FOR INITIAL TREATMENT OF MALIGNANT ASTROCYTOMA AND GLIOBLASTOMA MULTIFORME. Southern Medical Journal 1991, 84(9):2S42-42S43.

109. Gutin P, Wara W, Larson D, Leibel S, Prados M, Levin V, Sneed P, Weaver K, Silver P, et al: STEREOTAXIC INTERSTITIAL IODINE-125 BOOST IN THE INITIAL MANAGEMENT OF MALIGNANT GLIOMAS. International Journal of Radiation Oncology, Biology, Physics 1990, 19(SUPPL. 1):204.

110. Gutin PH, Leibel SA, Wara WM, Choucair A, Levin VA, Philips TL, Silver $P$, Dasilva V, Edwards MSB, Davis RL, et al: RECURRENT MALIGNANT GLIOMAS - SURVIVAL FOLLOWING INTERSTITIAL BRACHYTHERAPY WITH HIGHACTIVITY I-125 SOURCES. J Neurosurg 1987, 67(6):864-873.

111. Leibel S, Gutin P, Phillips T, Wara W, Choucair A, Weaver K, Lamb S, Silver P, Barnett C: IMPROVED SURVIVAL FOLLOWING INTERSTITIAL IMPLANTATION OF REMOVABLE HIGH-ACTIVITY IODINE-125 SOURCES FOR MALIGNANT RECURRENT GLIOMAS. International Journal of Radiation Oncology, Biology, Physics 1986, 12(SUPPL. 1):146.

112. Prados MD, Gutin PH, Phillips TL, Wara WM, Sneed PK, Larson DA, Lamb SA, Ham B, Malec MK, Wilson CB: INTERSTITIAL BRACHYTHERAPY FOR NEWLY DIAGNOSED PATIENTS WITH MALIGNANT GLIOMAS - THE UCSF EXPERIENCE. International Journal of Radiation Oncology Biology Physics 1992, 24(4):593-597.

113. Scharfen CO, Sneed PK, Wara WM, Larson DA, Phillips TL, Weaver KA, Prados MD, Gutin PH: STEREOTAXIC IODINE-125 BRAIN IMPLANTS FOR 
MALIGNANT GLIOMAS. International Journal of Radiation Oncology, Biology, Physics 1991, 21(SUPPL. 1):119.

114. Sneed PK, Gutin PH, Stauffer PR, Phillips TL, Prados MD, Weaver KA, Suen S, Lamb SA, Ham B, Ahn DK, et al: THERMORADIOTHERAPY OF RECURRENT MALIGNANT BRAIN-TUMORS. International Journal of Radiation Oncology Biology Physics 1992, 23(4):853-861.

115. Yakar D, Zamorano L, Dujovny M, Sheehan M, Kim JH: Interstitial iodine125 irradiation for malignant brain tumors: Advantage of permanent implant (PI) over temporary implants (TI). International Journal of Radiation Oncology Biology Physics 1992, 24(SUPPL. 1):143.

116. Fernandez P, Zamorano L, Gaspar L, Yakar D, Heilbrun LK: Permanent 125 I implant therapy with concurrent external beam radiation in the up-front treatment of malignant gliomas. Stereotactic and Functional Neurosurgery 1994, 63(1-4):287-288.

117. Fernandez PM, Zamorano L, Yakar D, Gaspar L, Warmelink C: PERMANENT I125 IMPLANTS IN THE UP-FRONT TREATMENT OF MALIGNANT GLIOMAS. Neurosurgery 1995, 36(3):467-473.

118. Fontanesi J, Clark WC, Weir A, Barry A, Kumar P, Miller A, Eddy T, Tai D, KUn LE: INTERSTITIAL I-125 AND CONCOMITANT CISPLATIN FOLLOWED BY HYPERFRACTIONATED EXTERNAL-BEAM IRRADIATION FOR MALIGNANT SUPRATENTORIAL GLIOMA - PRELIMINARY EXPERIENCE AT THE UNIVERSITY-OF-TENNESSEE, MEMPHIS. American Journal of Clinical Oncology-Cancer Clinical Trials 1993, 16(5):412-417.

119. Gaspar L, Zamorano L, Garcia L, Shamsa F, Warmelink C, Yakar D: Malignant gliomas: Permanent iodine-125 implants. International Journal of Radiation Oncology Biology Physics 1994, 30(SUPPL 1):295.

120. Schwarz SB, Siefert A, Ganswindt U, Kunz M, Kreth FW, Tonn JC, Belka C: The combination of percutaneous radiotherapy and (125)iodine-seedimplantation within the framework of multi model therapy concepts for cerebral tumours. Strahlentherapie Und Onkologie 2009, 185:84-84

121. Videtic GMM, Gaspar LE, Zamorano L, Fontanesi J, Levin KJ, Kupsky WJ, Tekyi-Mensah S: Use of the RTOG recursive partitioning analysis to validate the benefit of iodine-125 implants in the primary treatment of malignant gliomas. International Journal of Radiation Oncology Biology Physics 1999, 45(3):687-692.

122. Videtic GMM, Gaspar LE, Zamorano L, Stitt LW, Fontanesi J, Levin KJ: Implant volume as a prognostic variable in brachytherapy decisionmaking for malignant gliomas stratified by the RTOG recursive partitioning analysis. International Journal of Radiation Oncology Biology Physics 2001, 51(4):963-968.

123. Zamorano L, Gaspar L, Garcia L, Yakar D, Shamsa F, Buciuc R, Diaz FG: PERMANENT I-125 IMPLANT IN PRIMARY MALIGNANT GLIOMA. J Neurosurg 1995, 82(2):A350-A350.

124. Zamorano L, Li QH, Tekyi-Mensah S, Gaspar L, Fontanesi J, Levin K: Permanent iodine- 125 interstitial radiation therapy in the treatment of non-glioblastoma multiforme high-grade gliomas. Stereotactic and Functional Neurosurgery 2003, 81(1-4):10-17.

125. Hitchon PW, Vangilder JC, Wen BC, Jani S, Wetjen B, Sawin PD: Brachytherapy in malignant glioma. Stereotactic and Functional Neurosurgery 1994, 63(1-4):286-287.

126. Ryken TC, Hitchon PW, Vangilder JC, Wen BC, Jani S: Interstitial brachytherapy versus cytoreductive surgery in recurrent malignant glioma. Stereotactic and Functional Neurosurgery 1994, 63(1-4):241-245.

127. Malkin MG, Arbit E, Fass DE, Anderson LL, Osian A, Rosenblum MK, Shapiro JR, Leibel SA, Shapiro WR: STEREOTAXIC IMPLANTATION OF REMOVABLE HIGH-ACTIVITY IODINE-125 SOURCES FOR THE TREATMENT OF MALIGNANT GLIOMA. Neurology 1989, 39(3 SUPPL. 1):263.

128. Halligan JB, Stelzer KJ, Rostomily RC, Spence AM, Griffin TW, Berger MS: Operation and permanent low activity I-125 brachytherapy for recurrent high-grade astrocytomas. International Journal of Radiation Oncology Biology Physics 1996, 35(3):541-547.

129. Wick W, Hartmann C, Engel C, Stoffels M, Felsberg J, Stockhammer F, Sabel MC, Koeppen S, Ketter R, Meyermann R, et al: NOA-04 randomized phase III trial of sequential radiochemotherapy of anaplastic glioma with procarbazine, lomustine, and vincristine or temozolomide. J Clin Oncol 2009, 27(35):5874-5880.

130. Chen AM, Chang S, Pouliot J, Sneed PK, Prados MD, Lamborn KR, Malec MK, McDermott MW, Berger MS, Larson DA: Phase I trial of gross total resection, permanent iodine-125 brachytherapy, and hyperfractionated radiotherapy for newly diagnosed glioblastoma multiforme. International Journal of Radiation Oncology Biology Physics 2007, 69:825-830.
131. Darakchiev BJ, Albright RE, Breneman JC, Warnick RE: Safety and efficacy of permanent iodine-125 seed implants and carmustine wafers in patients with recurrent glioblastoma multiforme. J Neurosurg 2008, 108(2):236-242.

132. Keole S, Martinez J, Levin KJ, Frazier AJ, Zamorano L, Sloan A, Forman JD, Fontanesi JN: Survival analysis of patients with Glioblastoma multiforme treated with external radiotherapy followed by gamma knife radiosurgery or permanent lodine-125 implant boost. International Journal of Radiation Oncology Biology Physics 2001, 51(3 Supplement 1):256.

133. Larson DA, Suplica JM, Chang SM, Lamborn KR, McDermott MW, Sneed PK, Prados MD, Wara WM, Nicholas MK, Berger MS: Permanent iodine 125 brachytherapy in patients with progressive or recurrent glioblastoma multiforme. Neuro-Oncology 2004, 6(2):119-126.

134. Loeffler JS, Alexander E, Wen PY, Shea WM, Coleman CN, Kooy HM, Fine HA, Nedzi LA, Silver B, Riese NE, et al: RESULTS OF STEREOTAXIC BRACHYTHERAPY USED IN THE INITIAL MANAGEMENT OF PATIENTS WITH GLIOBLASTOMA. J Natl Cancer I 1990, 82(24):1918-1921.

135. Loeffler JS, Wen P, Alexander E III, Shea M, Black PM: RESULTS OF STEREOTACTIC INTERSTITIAL IRRADIATION USED IN TREATMENT OF RECURRENT GLIOBLASTOMA. Neurology 1991, 41(3 SUPPL. 1):411.

136. Sneed PK, Gutin PH, Larson DA, Malec MK, Phillips TL, Prados MD, Scharfen CO, Weaver KA, Wara WM: PATTERNS OF RECURRENCE OF GLIOBLASTOMA-MULTIFORME AFTER EXTERNAL IRRADIATION FOLLOWED BY IMPLANT BOOST. International Journal of Radiation Oncology Biology Physics 1994, 29(4):719-727.

137. Wen PY, Alexander E, Black PM, Fine HA, Riese N, Levin JM, Coleman CN, Loeffler JS: LONG-TERM RESULTS OF STEREOTAXIC BRACHYTHERAPY USED IN THE INITIAL TREATMENT OF PATIENTS WITH GLIOBLASTOMAS. Cancer 1994, 73(12):3029-3036.

138. Chamberlain MC, Kormanik P: Concurrent carboplatin and iodine-125 brachytherapy for recurrent glioblastoma multiforme. Ann Neurol 1997, 42(3):M152-M152.

139. Koot RW, Maarouf M, Hulshof M, Voges J, Treuer H, Koedooder C, Sturm V, Bosch DA: Brachytherapy - Results of two different therapy strategies for patients with primary glioblastoma multiforme. Cancer 2000, 88(12):2796-2802.

140. Patel S, Breneman JC, Warnick RE, Albright RE, Tobler WD, van Loveren HR, Tew JM: Permanent iodine-125 interstitial implants for the treatment of recurrent glioblastoma multiforme. Neurosurgery 2000, 46(5):1123-1128.

141. Sneed PK, Prados MD, McDermott MW, Larson DA, Malec MK, Lamborn KR, Davis RL, Weaver KA, Wara WM, Phillips TL, et al: LARGE EFFECT OF AGE ON THE SURVIVAL OF PATIENTS WITH GLIOBLASTOMA TREATED WITH RADIOTHERAPY AND BRACHYTHERAPY ROOST. Neurosurgery 1995, 36(5):898-903

142. Suplica JM, Berger MS, McDermott MW, Chang SM, Prados MD, Sneed PK, Tsao MN, Wara WM, Larson DA: Total resection and permanent high-dose I-125 brachytherapy for recurrent or progressive glioblastoma. International Journal of Radiation Oncology Biology Physics 1999, 45(3 SUPPL.):321.

143. Heideman RL, Douglass EC, Krance RA, Fontanesi J, Langston JA, Sanford RA, Kovnar EH, Ochs J, Kuttesch J, Jenkins JJ, et al: HIGH-DOSE CHEMOTHERAPY AND AUTOLOGOUS BONE-MARROW RESCUE FOLLOWED BY INTERSTITIAL AND EXTERNAL-BEAM RADIOTHERAPY IN NEWLY-DIAGNOSED PEDIATRIC MALIGNANT GLIOMAS. Journal of Clinical Oncology 1993, 11(8):1458-1465

144. Kitchen ND, Hughes SW, Taub NA, Sofat A, Beaney RP, Thomas DGT: SURVIVAL FOLLOWING INTERSTITIAL BRACHYTHERAPY FOR RECURRENT MALIGNANT GLIOMA. J Neuro-Oncol 1994, 18(1):33-39.

145. Sneed PK, Gutin PH, Prados MD, Phillips TL, Weaver KA, Wara WM, Larson DA: BRACHYTHERAPY OF BRAIN-TUMORS. Stereotactic and Functional Neurosurgery 1992, 59(1-4):157-165.

146. Stupp R, Hegi ME, Mason WP, van den Bent MJ, Taphoorn MJB, Janzer RC Ludwin SK, Allgeier A, Fisher B, Belanger K, et al: Effects of radiotherapy with concomitant and adjuvant temozolomide versus radiotherapy alone on survival in glioblastoma in a randomised phase III study: 5-year analysis of the EORTC-NCIC trial. Lancet Oncol 2009, 10(5):459-466.

147. Combs S, Debus J, Schulz-Ertner D: Radiotherapeutic alternatives for previously irradiated recurrent gliomas. BMC Cancer 2007, 7:167.

148. Chavantes MC, Zamorano L, Dujovny M, Milik G, Ausman Jl: A NEW APPROACH FOR BRAIN METASTASES FROM LUNG CANCER IMAGEGUIDED STEREOTAXIS AND IODINE-125 BRACHYTHERAPY. Chest 1988, 94(1 SUPPL):85S. 
149. Ruge Ml, Kocher M, Maarouf M, Hamisch C, Treuer H, Voges J, Sturm V: Comparison of Stereotactic Brachytherapy ((125)lodine Seeds) with Stereotactic Radiosurgery (LINAC) for the Treatment of Singular Cerebral Metastases. Strahlentherapie Und Onkologie 2011, 187(1):7-14.

150. Ostertag CB, Kreth FW: INTERSTITIAL I-125 RADIOSURGERY FOR CEREBRAL METASTASES. British Journal of Neurosurgery 1995, 9(5):593-603.

151. Schulder M, Black PM, Shrieve DC, Alexander E, Loeffler JS: Permanent lowactivity iodine-125 implants for cerebral metastases. J Neuro-Oncol 1997, 33(3):213-221

152. Alesch F, Hawliczek R, Koos WT: Interstitial irradiation of brain metastases. Acta Neurochirurgica Supplement; Stereotactic neuro-radio-surgery 1995, 29-34

153. Huang K, Sneed PK, Berger MS, Kunwar S, Larson DA, Wara WM, McDermott MW: Permanent iodine-125 brachytherapy for brain metastasis resection cavities. International Journal of Radiation Oncology Biology Physics 2002, 54(2 Supplement):238.

154. Ruge MI, Suchorska B, Maarouf M, Runge M, Treuer H, Voges J, Sturm V: Stereotactic (125)lodine Brachytherapy for the Treatment of Singular Brain Metastases: Closing a Gap? Neurosurgery 2011, 68(5):1209-1218.

155. Dagnew E, Kanski J, McDermott MW, Sneed PK, McPherson C, Breneman JC, Warnick RE: Management of newly diagnosed single brain metastasis using resection and permanent iodine- 125 seeds without initial wholebrain radiotherapy: a two institution experience. Neurosurg Focus 2007, 22(3):E3.

156. Huang K, Sneed PK, Kunwar S, Kragten A, Larson DA, Berger MS, Chan A, Pouliot J, McDermott MW: Surgical resection and permanent iodine-125 brachytherapy for brain metastases. J Neuro-Oncol 2009, 91(1):83-93.

157. Petr MJ, McPherson CM, Breneman JC, Warnick RE: Management of newly diagnosed single brain metastasis with surgical resection and permanent I-125 seeds without upfront whole brain radiotherapy. J Neuro-Oncol 2009, 92(3):393-400.

158. Prados M, Leibel S, Barnett CM, Gutin P: INTERSTITIAL BRACHYTHERAPY FOR METASTATIC BRAIN-TUMORS. Cancer 1989, 63(4):657-660.

159. Yakar D, Zamorano L, Dujovny M, Cooper M, Grandhe R, Martin F, Sheehan M: INTERSTITIAL TEMPORARY IMPLANTATION OF HIGH ACTIVITY IODINE-125 SOURCES FOR MALIGNANT GLIOMA AND BRAIN METASTASES. International Journal of Radiation Oncology, Biology, Physics 1989, 17(SUPPL. 1):228

160. Zamorano L, Yakar D, Dujovny M, Sheehan M, Kim J: PERMANENT I-125 IMPLANT AND EXTERNAL BEAM RADIATION-THERAPY FOR THE TREATMENT OF MALIGNANT BRAIN-TUMORS. Stereotactic and Functional Neurosurgery 1992, 59(1-4):183-192.

161. Zamorano L, Yakar D, Dujovny M, Sheehan M, Kim JH: Interstitial temporary implantation of high-activity iodine-125 sources for malignant glioma and brain metastases. Stereotactic and Functional Neurosurgery 1992, 59(1-4):182.

162. Kreth FW, Warnke PC, Ostertag CB: STEREOTAXIC INTERSTITIAL RADIOSURGERY AND PERCUTANEOUS RADIOTHERAPY FOR TREATMENT OF CEREBRAL METASTASES. Nervenarzt 1993, 64(2):108-113.

163. Ruge Ml, Kickingereder $P$, Grau S, Hoevels M, Treuer H, Sturm V: Stereotactic biopsy combined with stereotactic (125)iodine brachytherapy for diagnosis and treatment of locally recurrent single brain metastases. J Neurooncol 2011, 105(1):109-118.

164. Barlas O, Bayindir C, Imer M, Ayan I, Darendeliler E: Non-resective management of pineoblastoma. Minimally Invasive Neurosurgery 2000, 43(3):163-170.

165. Maarouf M, El Majdoub F, Buhrle C, Voges J, Lehrke R, Kocher M, Hunsche S, Treuer H, Sturm V: Pineal Parenchymal Tumors Management with Interstitial lodine-125 Radiosurgery. Strahlentherapie Und Onkologie 2010, 186(3):127-134

166. Kumar PP, Patil AA, Leibrock LG, Chu WK, Syh HW, McCaul GF, Reeves MA CONTINUOUS LOW-DOSE RATE BRACHYTHERAPY WITH HIGH-ACTIVITY I125 SEEDS IN THE MANAGEMENT OF MENINGIOMAS. International Journal of Radiation Oncology Biology Physics 1993, 25(2):325-328.

167. Kumar PP, Patil AA, Leibrock LG, Chu WK, Syh J, McCaul GF, Reeves MA: BRACHYTHERAPY - A VIABLE ALTERNATIVE IN THE MANAGEMENT OF BASAL MENINGIOMAS. Neurosurgery 1991, 29(5):676-680.

168. Kumar PP, Patil AA, Syh HW, Chu WK, Reeves MA: ROLE OF BRACHYTHERAPY IN THE MANAGEMENT OF THE SKULL BASE MENINGIOMA - TREATMENT OF SKULL BASE MENINGIOMAS. Cancer 1993, 71(11):3726-3731
169. Obasi PC, Barnett GH, Suh JH: Brachytherapy for intracranial meningioma using a permanently implanted iodine-125 seed. Stereotactic and Functional Neurosurgery 2002, 79(1):33-43.

170. Patil AA, Kumar P, Leibrock LG: Response of extra-axial tumors to stereotactically implanted high-activity $1-125$ seeds. Stereotactic and Functional Neurosurgery 1995, 64(3):139-152.

171. Vuorinen V, Heikkonen J, Brander A, Setala K, Sane T, Randell T, Paetau A, Pohjola J, Mantyla M, Jaaskelainen J: Interstitial radiotherapy of 25 parasellar/clival meningiomas and 19 meningiomas in the elderly Analysis of short-term tolerance and responses. Acta Neurochir 1996 138(5):495-508.

172. Kumar PP, Good RR, Cox TA, Leibrock LG, Skultety FM: REVERSAL OF VISUAL IMPAIRMENT AFTER INTERSTITIAL IRRADIATION OF PITUITARYTUMOR. Neurosurgery 1986, 18(1):82-84

173. Kumar PP, Good RR, Skultety FM, Leibrock LG, Severson GS: RADIATIONINDUCED NEOPLASMS OF THE BRAIN. Cancer 1987, 59(7):1274-1282.

174. Kumar PP, Good RR, Skultety FM, Leibrock LG: LOCAL-CONTROL OF RECURRENT CLIVAL AND SACRAL CHORDOMA AFTER INTERSTITIAL IRRADIATION WITH I-125 - NEW TECHNIQUES FOR TREATMENT OF RECURRENT OR UNRESECTABLE CHORDOMAS. Neurosurgery 1988, 22(3):479-483

175. Kumar PP: HIGH ACTIVITY IODINE-125 ENDOCURIETHERAPY FOR SKULL BASE TUMORS. International Journal of Radiation Oncology, Biology, Physics 1990, 19(SUPPL. 1):226

176. Kumar PP, Good RR, Leibrock LG, Mawk JR, Yonkers AJ, Ogren FP: HIGHACTIVITY IODINE 125 ENDOCURIETHERAPY FOR RECURRENT SKULL BASE TUMORS. Cancer 1988, 61(8):1518-1527.

177. Collins SP, Coppa ND, Zhang Y, Collins BT, McRae DA, Jean WC: CyberKnife (R) radiosurgery in the treatment of complex skull base tumors: analysis of treatment planning parameters. Radiation Oncology 2006, 1.

178. Minniti G, Amichetti M, Enrici RM: Radiotherapy and radiosurgery for benign skull base meningiomas. Radiat Oncol 2009, 4:42.

179. El Majdoub F, Brunn A, Berthold F, Sturm V, Maarouf M: Stereotaktische interstitielle Radiochirurgie zur Behandlung des intrakraniellen RosaiDorfman-Syndroms. Ein neuer therapeutischer Ansatz. Strahlentherapie Und Onkologie 2009, 185(2):109-112.

180. Suarez JC, Viano JC, Zunino S, Herrera EJ, Gomez J, Tramunt B, Marengo I, Hiramatzu E, Miras M, Pena M, et al: Management of child optic pathway gliomas: new therapeutical option. Childs Nervous System 2006, 22(7):679-684.

181. Barlas O, Bayindir C, Can M: Interstitial irradiation for craniopharyngioma. Acta Neurochir 2000, 142(4):389-395

182. Frank F, Fabrizi AP, Frank G, Fioravanti A: Stereotactic management of craniopharyngiomas. Stereotactic and Functional Neurosurgery 1995, 65(14):176-183

183. El Majdoub F, Simon T, Hoevels M, Berthold F, Sturm V, Maarouf M: Interstitial Brachytherapy using Stereotactic Implanted 125lodine Seeds for Recurrent Medulloblastoma. Clinical Oncology , Corrected Proof

184. Chalifoux R, Elisevich K: Effect of ionizing radiation on partial seizures attributable to malignant cerebral tumors. Stereotactic and Functional Neurosurgery 1996, 67(3-4):169-182.

185. Chamberlain MC, Barba D, Kormanik P, Berson AM, Saunders WM, Shea MC: CONCURRENT CISPLATIN THERAPY AND IODINE-125 BRACHYTHERAPY FOR RECURRENT MALIGNANT BRAIN-TUMORS. Archives of Neurology 1995, 52(2):162-167

186. Tacke U, Karger D, Spreer J, Berlis A, Nikkhah G, Korinthenberg R: Incidence of vasculopathy in children with hypothalamic/chiasmatic gliomas treated with brachytherapy. Childs Nervous System 2011, 27(6):961-966.

187. Bampoe J, Nag S, Leung P, Laperriere N, Bernstein M: Brain necrosis after permanent low-activity iodine-125 implants: case report and review of toxicity from focal radiation. Brain Tumor Pathol 2000, 17(3):139-145.

188. Agbi CB, Bernstein M, Laperriere N, Leung P, Lumley M: PATTERNS OF RECURRENCE OF MALIGNANT ASTROCYTOMA FOLLOWING STEREOTAXIC INTERSTITIAL BRACHYTHERAPY WITH I-125 IMPLANTS. International Journal of Radiation Oncology Biology Physics 1992, 23(2):321-326.

189. Wara WM: RADIATION-THERAPY FOR BRAIN-TUMORS. Cancer 1985 55(9):2291-2295.

190. Tihan T, Barletta J, Parney I, Lamborn K, Sneed PK, Chang S: Prognostic value of detecting recurrent glioblastoma multiforme in surgical specimens from patients after radiotherapy: should pathology 
evaluation atter treatment decisions? Human Pathology 2006, 37(3):272-282.

191. Frank F, Gaist G, Piazza G, Ricci RF, Sturiale C, Galassi E: STEREOTAXIC BIOPSY AND RADIOACTIVE IMPLANTATION FOR INTERSTITIAL THERAPY OF TUMORS OF THE PINEAL REGION. Surgical Neurology 1985, 23(3):275-280

192. Houston SC, Crocker IR, Brat DJ, Olson JJ: Extraneural metastatic glioblastoma after interstitial brachytherapy. International Journal of Radiation Oncology Biology Physics 2000, 48(3):831-836.

193. Correa CF, Teixeira MJ, Rogano LA, Oliveira S, Miziara M, Gusmao C, Bouza AA: 125 I brachytherapy for treatment of CNS tumors. Stereotactic and Functional Neurosurgery 1996, 67(1-2):93.

194. Leibel SA, Gutin PH, Phillips TL, Wara WM, Weaver KA, Lamb S: INTERSTITIAL IMPLANTATION OF HIGH-ACTIVITY IODINE-125 SOURCES FOR THE TREATMENT OF RECURRENT MALIGNANT BRAIN TUMORS. International Journal of Radiation Oncology, Biology, Physics 1984, 10(SUPPL. 2):144-145.

195. Leibel SA, Gutin PH, Phillips TL, Wara WM, Weaver KA, Lamb S: INTERSTITIAL BRACHYTHERAPY WITH REMOVABLE HIGH-ACTIVITY I-125 SOURCES FOR RECURRENT MALIGNANT BRAIN-TUMORS. American Journal of Clinical Oncology-Cancer Clinical Trials 1985, 8(1):20-20.

196. Vordermark D: Brachytherapy - Results of two different therapy strategies for patients with primary glioblastoma multiforme. Cancer 2001, 91(6):1185-1186.

197. Rostomily RC, Halligan J, Geyer R, Stelzer K, Lindsley K, Berger MS: Permanent low-activity $\mathrm{I}-125$ seed placement for the treatment of pediatric brain tumors: Preliminary experience. Pediatric Neurosurgery 2001, 34(4):198-205.

198. Korinthenberg RND, Trippel M, Ostertag C, Nikkhah G: Long-Term Results of Brachytherapy with temporary iodine-125 Seeds in Children with lowgrade gliomas. International Journal Radiation Oncology Biology Physics 2011, 79(4):1131-1138

199. Ruge MI, Simon T, Suchorska B, Lehrke R, Hamisch C, Koerber F, Maarouf M, Treuer H, Berthold F, Sturm V, et al: Stereotactic brachytherapy with iodine-125 seeds for the treatment of inoperable low-grade gliomas in children: long-term outcome. J Clin Oncol 2011, 29(31):4151-4159.

doi:10.1186/1748-717X-7-30

Cite this article as: Schwarz et al.: lodine-125 brachytherapy for brain tumours - a review. Radiation Oncology 2012 7:30.

\section{Submit your next manuscript to BioMed Central and take full advantage of:}

- Convenient online submission

- Thorough peer review

- No space constraints or color figure charges

- Immediate publication on acceptance

- Inclusion in PubMed, CAS, Scopus and Google Scholar

- Research which is freely available for redistribution

Submit your manuscript at www.biomedcentral.com/submit
Biomed Central 